\title{
Large-size expansion for triangular Wilson loops in confining gauge theories
}

\section{P.V. Pobylitsa}

Petersburg Nuclear Physics Institute, Gatchina, 188300, St. Petersburg, Russia

E-mail: pp9@gmx.de

Abstract: The asymptotic behavior of Wilson loops in the large-size limit $(L \rightarrow \infty)$ in confining gauge theories with area law is controlled by effective string theory (EST). The $L^{-2}$ term of the large-size expansion for the logarithm of Wilson loop appears within EST as a 2-loop correction. Ultraviolet divergences of this 2-loop correction for polygonal contours can be renormalized using an analytical regularization constructed in terms of Schwarz-Christoffel mapping. In the case of triangular Wilson loops this method leads to a simple final expression for the $L^{-2}$ term.

Keywords: Wilson, 't Hooft and Polyakov loops, Bosonic Strings

ARXIV EPRINT: 1908.01724 


\section{Contents}

1 Introduction 1

1.1 Large-size expansion for Wilson loops 1

1.2 Simple example 3

1.3 Known results for $f_{2}(C) \quad 4$

1.4 Outline of the work 5

2 Wilson loops in EST $\quad 6$

2.1 Expansion of Nambu action 6

$\begin{array}{lll}2.2 & \text { Loop expansion in EST } & 7\end{array}$

2.3 2-loop EST correction 9

3 Renormalization $\quad 11$

3.1 Rectangular case: method of K. Dietz and T. Filk 11

$\begin{array}{ll}3.2 & \text { General polygons: renormalization based on SC mapping } \\ 3.3\end{array}$

3.3 First step of renormalization: from $\left\langle\mathcal{S}_{4}\right\rangle$ to $\left\langle\mathcal{S}_{4}\right\rangle^{\text {ren,1 }} \quad 13$

4 Complex representation $\quad 14$

$\begin{array}{lll}4.1 & \text { Definitions and conventions } & 14\end{array}$

$\begin{array}{ll}4.2 \text { Conformal mapping to semiplane } & 15\end{array}$

$\begin{array}{lll}4.3 & \text { Reduction of }\left\langle\mathcal{S}_{4}\right\rangle^{\text {ren,1 }} \text { to basic integrals } I_{1} \text { and } I_{2} & 15\end{array}$

5 Polygons and SC mapping $\quad 16$

$\begin{array}{ll}5.1 \text { SC mapping with finite vertices } & 16\end{array}$

$\begin{array}{lll}5.2 & \mathrm{SL}(2, \mathbb{R}) \text { symmetry } & 17\end{array}$

$\begin{array}{lll}5.3 \text { SC mapping with one vertex at infinity } & 18\end{array}$

$\begin{array}{lll}5.4 \text { SC vertex at infinity: pro and contra } & 19\end{array}$

6 Analytical regularization $\quad 19$

$\begin{array}{lll}6.1 & \text { Functions } M_{P}^{(n)} & 19\end{array}$

6.2 From divergent integrals to meromorphic functions 21

$\begin{array}{lll}6.3 & \text { Functions } \Pi_{P}^{(n)} & 22\end{array}$

7 Calculation of $f_{2}(C)$ for triangles $\quad 22$

$\begin{array}{lll}7.1 & \text { Case of triangles } & 22\end{array}$

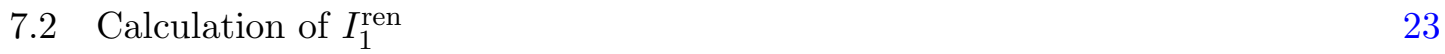

$\begin{array}{ll}7.3 \text { Calculation of } I_{2}^{\text {ren }} & 23\end{array}$

$\begin{array}{lll}7.4 & \text { Final result } & 25\end{array}$

8 Conclusions $\quad 26$ 
A Properties of Laplace determinants

A.1 Heat-kernel expansion for Laplace operator 26

$\begin{array}{lll}\text { A.2 Renormalization of Laplace determinants } & 27\end{array}$

$\begin{array}{ll}\text { B 1-loop EST corrections } & 27\end{array}$

C Calculation of Laplace determinants for polygons $\quad 29$

$\begin{array}{lll}\text { C.1 2D Laplace determinant for general polygons } & 29\end{array}$

C.1.1 Results of E. Aurell and P. Salomonson 29

$\begin{array}{lll}\text { C.1.2 SC mapping from unit circle to polygon } & 31\end{array}$

$\begin{array}{lll}\text { C.1.3 Result for Laplace determinant } & 31\end{array}$

C.1.4 SC mapping: from unit circle to semiplane 31

C.1.5 Laplace determinants in terms of semiplane parameters of SC mapping 32

C.1.6 Taking one SC vertex to infinity 32

$\begin{array}{lll}\text { C.1.7 Balanced combinations of Laplace determinants } & 32\end{array}$

C.1.8 Result for balanced linear combinations of $f_{0}\left(C_{a}\right) \quad 33$

C.2 Case of triangles 34

$\begin{array}{lll}\text { C.2.1 Result } & 34\end{array}$

C.2.2 Nontriviality of vertex-balance condition for triangles 34

$\begin{array}{lll}\text { C.2.3 Derivation } & 35\end{array}$

$\begin{array}{ll}\text { D Schwarz derivative } & 36\end{array}$

$\begin{array}{lr}\text { E Propagator in diagonal limit } & \mathbf{3 6}\end{array}$

E.1 Green function 36

$\begin{array}{lll}\text { E.2 } & \text { Limit } 1 & 37\end{array}$

$\begin{array}{lll}\text { E.3 } & \text { Limit } 2 & 38\end{array}$

$\begin{array}{lll}\text { F } & \text { SC mapping for triangles } & 39\end{array}$

G Calculation of $\Pi_{1}^{(2)} \quad 40$

H Calculation of $\Pi_{P}^{(2)}+42$

\section{Introduction}

\subsection{Large-size expansion for Wilson loops}

Gauge theories obeying area law [1]

$$
\lim _{|C| \rightarrow \infty} \frac{1}{S(C)} \ln W(C)=-\sigma \neq 0
$$

for Wilson loops

$$
W(C)=\left\langle\operatorname{Tr} \mathrm{P} \exp \left[i \oint_{C} d x^{\mu} A_{\mu}(x)\right]\right\rangle
$$


(with large flat contour $C$ bounding a region with area $S(C)$ ) play an important role as models of the heavy-quark confinement and are often briefly called confining gauge theories (although the problem of confinement in real QCD is more complicated). In our modern understanding of confining gauge theories, the area law is considered as a secondary manifestation of a much deeper phenomenon of effective string formation. This phenomenon is described by effective string theory (EST) which provides a detailed information about the asymptotic behavior of Wilson loops in the limit of large size of contour $C$ (denoted informally as $|C| \rightarrow \infty$ in eq. (1.1)). EST assumes that the asymptotic behavior of Wilson loops in the limit of large size of contour $C$ can be described by a functional integral over surfaces $\Sigma$ bounded by contour $C$ :

$$
W(C) \rightarrow \text { const } \int_{\partial \Sigma=C} D \Sigma \exp \left(-\mathcal{S}_{\mathrm{EST}}[\Sigma]\right) .
$$

The idea that Wilson loops $W(C)$ can be approximated by functional integral (1.3) has a long history. Starting from qualitative and heuristic arguments [2], one can try to justify the stringy approach to Wilson loops using various limits and expansions: large size, large number of colors [3-6], large number of space-time dimensions [7-10], Regge limit [10-12]. Here we are interested in EST understood as an effective theory describing the large-size limit. The first steps in this direction were made by M. Lüscher, G. Münster, K. Symanzik and P. Weisz [13-15]. In the computation of the first terms of the large-size expansion, one can approximate $\mathcal{S}_{\text {EST }}[\Sigma]$ by Nambu action

$$
\mathcal{S}_{\text {Nambu }}(\Sigma)=\sigma_{0} S(\Sigma)
$$

where $S(\Sigma)$ is the area of surface $\Sigma$ and $\sigma_{0}$ is the bare string tension (different from the renormalized physical string tension $\sigma$ appearing in area law (1.1)). If one wants to use EST for the calculation of higher-order terms of the large-size expansion then $S_{\mathrm{EST}}[\Sigma]$ must be understood as an infinite series containing all possible terms compatible with the symmetries of the problem.

The theoretical work in EST has been proceeding in various directions including

- derivation of general constraints on terms appearing in EST action $S_{\mathrm{EST}}[\Sigma]$ [16-25],

- computation of loop corrections in EST for rectangular Wilson loops [24, 26-29] and for other closely related quantities like correlation functions of Polyakov lines and spectra of closed and open strings [16, 18-23],

- analysis of string finite-width effects [15, 30, 31].

The predictions of EST have been successfully verified by many lattice Monte Carlo tests (see [24, 32-38] and references therein).

In the case when large-size limit $|C| \rightarrow \infty$ is implemented as a uniform rescaling of contour $C$ with a large factor $\lambda$, EST predicts the following structure of the large-size expansion for polygonal contours $C$ :

$$
\ln W(\lambda C) \stackrel{\lambda \rightarrow \infty}{=} f_{\ln }(C) \ln \lambda+\sum_{k \geq-2} \lambda^{-k} f_{k}(C) .
$$


Here $f_{k}(C)(k=\ln ,-2,-1,0, \ldots)$ are functionals of contour $C$. EST provides much information on $f_{k}(C)$ but some parameters controlling $f_{k}(C)$ depend on the underlying microscopic gauge theory (MGT).

Two leading terms of expansion (1.5) are associated with area $S(C)$ and perimeter $L(C)$ :

$$
\begin{aligned}
& f_{-2}(C)=-\sigma S(C), \\
& f_{-1}(C)=\rho L(C) .
\end{aligned}
$$

Here $\sigma$ is the string tension appearing in eq. (1.1). Parameters $\sigma, \rho$ are determined by MGT and cannot be computed in EST. Parameter $\rho$ depends on the renormalization scheme used for Wilson loops in MGT whereas string tension $\sigma$ is renormalization invariant in MGT.

Some properties of functionals $f_{k}(C)$ can be derived on general grounds without a direct involvement of EST. For example, the compatibility of expansion (1.5) with transitivity $W\left(\left(\lambda_{1} \lambda_{2}\right) C\right)=W\left(\lambda_{1}\left(\lambda_{2} C\right)\right)$ leads to relation

$$
f_{\ln }(C) \ln \left(\lambda_{1} \lambda_{2}\right)+\sum_{k \geq-2}\left(\lambda_{1} \lambda_{2}\right)^{-k} f_{k}(C)=f_{\ln }\left(\lambda_{2} C\right) \ln \lambda_{1}+\sum_{k \geq-2} \lambda_{1}^{-k} f_{k}\left(\lambda_{2} C\right)
$$

which results in

$$
\begin{aligned}
f_{k}(\lambda C) & =\lambda^{-k} f_{k}(C) \quad(k \neq 0), \\
f_{0}(\lambda C) & =f_{0}(C)+f_{\ln }(C) \ln \lambda, \\
f_{\ln }(\lambda C) & =f_{\ln }(C)
\end{aligned}
$$

Using EST, one can show that

$$
f_{1}(C)=0
$$

(see section 2.2).

\subsection{Simple example}

The main subject of this paper is functional $f_{2}(C)$. In the case of rectangular contours, $f_{2}(C)$ has been already computed (see section 1.3). Our aim is to provide a general computational scheme (including regularization and renormalization) for the case of $f_{2}(C)$ with arbitrary polygons $C$ and to perform an explicit calculation for the simplest case of triangles $C$.

Although term $\lambda^{-2} f_{2}(C)$ is strongly suppressed in expansion (1.5), this term plays a crucial role in lattice tests of EST. The computation of this correction for general polygons is also important for understanding the structure of renormalization in EST (in the sense of renormalization in effective theories).

As for more dominant terms of expansion (1.5) associated with $f_{\ln }(C)$ and $f_{0}(C)$, these terms are well-known. A brief review of their properties can be found in appendices B, C.

In this introductory section we would like to concentrate on functional $f_{2}(C)$ and to minimize the involvement of terms $f_{\ln }(C)$ and $f_{0}(C)$. Therefore it makes sense to start 
from an expression combining several Wilson loops in such a way that the role of term $f_{2}(C)$ is enhanced whereas some of dominant terms $f_{k}(C)$ cancel. An instructive example is ratio

$$
\frac{W\left(\lambda \nu_{1} C\right) W\left(\lambda \nu_{2} C\right)}{\left[W\left(\lambda \frac{\nu_{1}+\nu_{2}}{2} C\right)\right]^{2}}
$$

taken in the limit $\lambda \rightarrow \infty$ at fixed $\nu_{1}, \nu_{2}$. Using properties (1.9)-(1.12) of expansion (1.5), we find

$$
\begin{aligned}
\ln \frac{W\left(\lambda \nu_{1} C\right) W\left(\lambda \nu_{2} C\right)}{\left[W\left(\lambda \frac{\nu_{1}+\nu_{2}}{2} C\right)\right]^{2}} \stackrel{\lambda \rightarrow \infty}{=} & -\frac{1}{2} \lambda^{2}\left(\nu_{1}-\nu_{2}\right)^{2} \sigma S(C) \\
& +f_{\ln }(C) \ln \frac{4 \nu_{1} \nu_{2}}{\left(\nu_{1}+\nu_{2}\right)^{2}} \\
& +\frac{1}{\lambda^{2}}\left[\frac{1}{\nu_{1}^{2}}+\frac{1}{\nu_{2}^{2}}-\frac{8}{\left(\nu_{1}+\nu_{2}\right)^{2}}\right] f_{2}(C)+O\left(\lambda^{-3}\right) .
\end{aligned}
$$

The 1.h.s. is a renormalization invariant combination of Wilson loops (which can be computed, e.g. by lattice Monte Carlo simulations). On the r.h.s. we have an asymptotic series in inverse powers of $\lambda \rightarrow \infty$. Note that terms $f_{-1}(C)$ and $f_{0}(C)$ cancel on the r.h.s. of (1.14). The explicit expression for $f_{\ln }(C)$ is given by eq. (B.2).

Expansion (1.14) provides a good illustration of the role of the $\lambda^{-2} f_{2}(C)$ correction. On the other hand, one can construct more complicated renormalization invariant ratios of Wilson loops whose large-size expansions involve also $f_{0}(C)$ : see appendices B, C.

\subsection{Known results for $f_{2}(C)$}

Dimensional arguments and scaling property (1.9) lead to the following structure of $f_{2}(C)$ :

$$
f_{2}(C)=\frac{1}{\sigma S(C)} g_{2}(C)
$$

where $\sigma$ is the string tension appearing in area law (1.1) and $g_{2}(C)$ is a dimensionless function depending only on the geometry of polygon $C$.

For rectangular contours, $f_{2}(C)$ was computed in refs. [26, 27] with an unfortunate arithmetic error which was later detected and corrected in refs. [28, 29]. For rectangle $C_{\text {rectangle }}\left(L_{1}, L_{2}\right)$ with sides $L_{1}, L_{2}$ one has

$$
\begin{aligned}
g_{2}\left(C_{\text {rectangle }}\left(L_{1}, L_{2}\right)\right)=\left(\frac{\pi}{24}\right)^{2}(D-2)[ & 2\left(\frac{L_{1}}{L_{2}}\right)^{2} E_{4}\left(i \frac{L_{1}}{L_{2}}\right) \\
& \left.-\frac{D-6}{2} E_{2}\left(i \frac{L_{1}}{L_{2}}\right) E_{2}\left(i \frac{L_{2}}{L_{1}}\right)\right]
\end{aligned}
$$

where $D$ is the space-time dimension and $E_{n}(z)$ are Eisenstein series.

In this work we compute $f_{2}(C)$ for the case of triangular loops $C$. For triangle $C_{\text {triangle }}\left(\theta_{1}, \theta_{2}, \theta_{3} ; S\right)$ with interior angles $\theta_{1}, \theta_{2}, \theta_{3}$ and with area $S$ we find (see section 7.4)

$$
g_{2}\left(C_{\text {triangle }}\left(\theta_{1}, \theta_{2}, \theta_{3} ; S\right)\right)=\frac{1}{8} Y(1-Y)
$$


where

$$
Y=\frac{D-2}{24} \prod_{k=1}^{3}\left(\frac{\pi}{\theta_{k}}-1\right) .
$$

According to eq. (1.15), the full explicit expression for $f_{2}\left(C_{\text {triangle }}\right)$ is

$$
\begin{aligned}
f_{2}\left(C_{\text {triangle }}\left(\theta_{1}, \theta_{2}, \theta_{3} ; S\right)\right)= & \frac{1}{\sigma S} \frac{D-2}{192} \\
& \times\left[\prod_{k=1}^{3}\left(\frac{\pi}{\theta_{k}}-1\right)\right]\left[1-\frac{D-2}{24} \prod_{k=1}^{3}\left(\frac{\pi}{\theta_{k}}-1\right)\right] .
\end{aligned}
$$

\subsection{Outline of the work}

The main part of this work concentrates on the computation of $f_{2}(C)$ in large-size expansion (1.5). Working with ratios (1.14) of Wilson loops, one can eliminate more dominant term $f_{0}(C)$ from the consideration. Nevertheless for completeness a review of well-known results for terms $f_{\ln }(C)$ and $f_{0}(C)$ is provided in appendices $\mathrm{A}, \mathrm{B}, \mathrm{C}$.

In section 2 we sketch basic formulas of the loop expansion in EST. This part of the work is common for our triangular case and for the rectangular case studied in refs. [26, 27]. Correction $f_{2}(C)$ is given by a figure-eight-like Feynman diagram for a two-dimensional field theory of $(\partial \phi)^{2}+(\partial \phi)^{4}$ type (see eq. (2.7)) in the region bounded by contour $C$ with zero boundary conditions. We use notation $\left\langle\mathcal{S}_{4}\right\rangle$ (see eqs. (2.11), (2.17)) for the integral associated with this Feynman diagram. In principle, $\left\langle\mathcal{S}_{4}\right\rangle$ represents the result for $f_{2}(C)$ (up to the sign). However, this Feynman diagram has ultraviolet divergences.

The deviation of the current work from refs. $[26,27]$ starts when it comes to the regularization of ultraviolet divergences in $\left\langle\mathcal{S}_{4}\right\rangle$. Unfortunately the regularization used in refs. $[26,27]$ can be applied only to the case of rectangular contours $C$. In our work we suggest another regularization which can be used for arbitrary polygons. In section 3 we describe the difference between the regularization used in refs. [26, 27] and our regularization. Our renormalization procedure operationally consists of two steps:

$$
\left\langle\mathcal{S}_{4}\right\rangle \stackrel{\text { step }}{\longrightarrow}\left\langle\mathcal{S}_{4}\right\rangle^{\text {ren,1 } 1} \stackrel{\text { step } 2}{\longrightarrow}\left\langle\mathcal{S}_{4}\right\rangle^{\text {ren }} \equiv-f_{2}(C) .
$$

Here $\left\langle\mathcal{S}_{4}\right\rangle^{\text {ren }}$ is completely free of ultraviolet divergences and provides the result for $f_{2}(C)$ (see eq. (2.24)) whereas the intermediate quantity $\left\langle\mathcal{S}_{4}\right\rangle^{\text {ren,1 }}$ (introduced in eq. (3.12)) is only partly renormalized and still has ultraviolet divergences.

Our calculation is based on Schwarz-Christoffel (SC) mapping for polygon $C$ and on other powerful methods of complex analysis. In section 4 we introduce a complexified representation for $\left\langle\mathcal{S}_{4}\right\rangle$ and use it in order to perform the first step of renormalization $\left\langle\mathcal{S}_{4}\right\rangle \rightarrow\left\langle\mathcal{S}_{4}\right\rangle^{\text {ren,1 }}$ (with technical details placed in appendix E).

In section 5 the derived expression for $\left\langle\mathcal{S}_{4}\right\rangle^{\text {ren,1 }}$ is rewritten in terms of SC mapping.

In section 6 we describe the second step of renormalization procedure $\left\langle\mathcal{S}_{4}\right\rangle^{\text {ren, } 1} \rightarrow\langle S\rangle^{\text {ren }}$ based on the analytical regularization of the SC representation for $\left\langle\mathcal{S}_{4}\right\rangle^{\text {ren,1 }}$, assuming the general case of arbitrary polygons $C$. 
In section 7 we apply the general formula derived for $\langle S\rangle^{\text {ren }}$ with an arbitrary polygon to the case of triangles. We show that in the triangular case all integrals involved in the computation of $\langle S\rangle^{\text {ren }}$ can be computed explicitly and derive final expression (1.19) for $f_{2}(C)=-\langle S\rangle^{\mathrm{ren}}$. The technical details of the computation are placed in appendices F, G, H. For completeness we also provide information about $f_{0}(C)$ with triangular contours $C$ in appendix C.2.

\section{Wilson loops in EST}

\subsection{Expansion of Nambu action}

The first steps of our work are identical to those of refs. [26, 27]. For the computation of the first orders of the large-size expansion of Wilson loops (including $f_{2}(C)$ ), one can replace full EST action $\mathcal{S}_{\mathrm{EST}}[\Sigma]$ in eq. (1.3) by Nambu action (non-Nambu terms of effective action $\mathcal{S}_{\mathrm{EST}}[\Sigma]$ become essential starting from the computation of correction $f_{3}(C)$ generated by the boundary action $[16,19,24])$. Thus for our aim we can approximate

$$
W(C) \rightarrow \text { const } \int_{\partial \Sigma=C} D \Sigma \exp \left[-\mathcal{S}_{\mathrm{Nambu}}(\Sigma)\right]
$$

with $\mathcal{S}_{\text {Nambu }}(\Sigma)$ given by eq. (1.4). Assuming parametrization $X^{a}\left(x^{1}, x^{2}\right)$ of surface $\Sigma$, one has

$$
\begin{aligned}
\mathcal{S}_{\text {Nambu }}(\Sigma) & =\sigma_{0} \int d x^{1} d x^{2} \sqrt{\operatorname{det}_{\mu \nu}\left(Q_{\mu \nu}\right)}, \\
Q_{\mu \nu} & =\sum_{a=1}^{D} \frac{\partial X^{a}}{\partial x^{\mu}} \frac{\partial X^{a}}{\partial x^{\nu}} .
\end{aligned}
$$

In EST (like in the theory of fundamental boson strings) the reparametrization invariance must be treated as a gauge symmetry. In the case of flat Wilson contours $C$ lying in the $X^{1}, X^{2}$ plane, it is convenient to use the planar gauge (sometimes called static gauge):

$$
\begin{array}{ll}
X^{a}\left(x^{1}, x^{2}\right)=x^{a} & (a=1,2), \\
X^{a}\left(x^{1}, x^{2}\right)=Y^{a}\left(x^{1}, x^{2}\right) & (a=3,4, \ldots, D) .
\end{array}
$$

Expanding up to quartic terms, we find in this gauge

$$
\begin{aligned}
\sqrt{\operatorname{det}_{\mu \nu}\left(Q_{\mu \nu}\right)}= & 1+\frac{1}{2} \sum_{\mu=1}^{2} \sum_{a=3}^{D}\left(\frac{\partial Y^{a}}{\partial x^{\mu}}\right)^{2} \\
& +\sum_{\mu, \nu=1}^{2} \sum_{a, b=3}^{D}\left[\frac{1}{8}\left(\frac{\partial Y^{a}}{\partial x^{\mu}}\right)^{2}\left(\frac{\partial Y^{b}}{\partial x^{\nu}}\right)^{2}-\frac{1}{4} \frac{\partial Y^{a}}{\partial x^{\mu}} \frac{\partial Y^{b}}{\partial x^{\mu}} \frac{\partial Y^{a}}{\partial x^{\nu}} \frac{\partial Y^{b}}{\partial x^{\nu}}\right]+\ldots
\end{aligned}
$$

We use notation $U(C)$ for the flat region in $x^{1}, x^{2}$ plane bounded by Wilson contour $C$. 
Thus

$$
\begin{aligned}
\frac{1}{\sigma_{0}} \mathcal{S}_{\mathrm{Nambu}}= & \int_{U(C)} d^{2} x \sqrt{\operatorname{det}_{\mu \nu}\left(Q_{\mu \nu}\right)} \approx S(C)+\frac{1}{2} \int_{U(C)} d^{2} x \sum_{\mu=1}^{2} \sum_{a=3}^{D}\left(\frac{\partial Y^{a}}{\partial x^{\mu}}\right)^{2} \\
& +\int_{U(C)} d^{2} x \sum_{\mu, \nu=1}^{2} \sum_{a, b=3}^{D}\left[\frac{1}{8}\left(\frac{\partial Y^{a}}{\partial x^{\mu}}\right)^{2}\left(\frac{\partial Y^{b}}{\partial x^{\nu}}\right)^{2}-\frac{1}{4} \frac{\partial Y^{a}}{\partial x^{\mu}} \frac{\partial Y^{b}}{\partial x^{\mu}} \frac{\partial Y^{a}}{\partial x^{\nu}} \frac{\partial Y^{b}}{\partial x^{\nu}}\right]+\ldots
\end{aligned}
$$

Here $S(C)$ is the area of region $U(C)$ bounded by flat contour $C$. One can rewrite this expansion in the form

$$
\mathcal{S}_{\mathrm{Nambu}}=\mathcal{S}_{0}+\mathcal{S}_{2}+\mathcal{S}_{4}+\ldots
$$

where

$$
\begin{aligned}
& \mathcal{S}_{0}=\sigma_{0} S(C) \\
& \mathcal{S}_{2}=\frac{1}{2} \sigma_{0} \int_{U(C)} d^{2} x \sum_{\mu=1}^{2} \sum_{a=3}^{D}\left(\frac{\partial Y^{a}}{\partial x^{\mu}}\right)^{2} \\
& \mathcal{S}_{4}=\sigma_{0} \int_{U(C)} d^{2} x \sum_{\mu, \nu=1}^{2} \sum_{a, b=3}^{D}\left[\frac{1}{8}\left(\frac{\partial Y^{a}}{\partial x^{\mu}}\right)^{2}\left(\frac{\partial Y^{b}}{\partial x^{\nu}}\right)^{2}-\frac{1}{4} \frac{\partial Y^{a}}{\partial x^{\mu}} \frac{\partial Y^{b}}{\partial x^{\mu}} \frac{\partial Y^{a}}{\partial x^{\nu}} \frac{\partial Y^{b}}{\partial x^{\nu}}\right] .
\end{aligned}
$$

\subsection{Loop expansion in EST}

The large-size expansion for Wilson loops is constructed by applying the steepest-descent method to functional integral (2.1). Expanding in small fluctuations of $\Sigma$ near the minimal surface spanned on contour $C$, one finds in the 1-loop approximation [13-15]:

$$
\begin{aligned}
\int_{\partial \Sigma=C} D \Sigma \exp \left[-\mathcal{S}_{\text {Nambu }}(\Sigma)\right]= & \text { const }\left[\operatorname{Det}_{\text {reg }}[-\Delta(C)]\right]^{-(D-2) / 2} \exp \left[-\sigma_{0} S(C)\right] \\
& \times\left[1+O\left(\frac{1}{\sigma_{0}}\right)\right]
\end{aligned}
$$

Here Det Deg $_{\text {re }}[-\Delta(C)]$ is the functional determinant of Laplace operator (referred as Laplace determinant for brevity) with zero (Dirichlet) boundary conditions on contour $C$ and with properly regularized ultraviolet divergences. In this oversimplified approach, at intermediate stages of the calculation we formally deal with the loop expansion in small parameter $1 / \sigma_{0} \rightarrow 0$ at fixed contour $C$ but in the end this formal $1 / \sigma_{0}$ expansion can be rearranged as a large-size expansion for Wilson loops at fixed physical (renormalized) string tension $\sigma$. The real situation is more complicated because

- loop corrections of EST have ultraviolet divergences which must be renormalized (in the sense of renormalization in effective theories),

- microscopic gauge theory (MGT) has its own ultraviolet divergences and renormalization. 
Fortunately, these complications play a limited role if one is interested in the EST computation of the first terms of large-size expansion (1.5).

For the computation of $f_{2}(C)$ one needs only the Nambu term of the full effective action. The loop expansion of EST with Nambu action naively goes in integer powers of $1 / \sigma_{0}$ which results in even terms $\lambda^{-2 n} f_{2 n}(C)$ of large-size expansion (1.5). This naive argument explains the vanishing of odd term $f_{1}(C)$ (1.12) but a serious proof of (1.12) requires more effort since divergences and their renormalization in EST may generate odd and non-analytical terms like $f_{-1}(C)$ and $f_{\ln }(C)$.

Anyway the concept of the naive and formal loop expansion in small parameter $1 / \sigma_{0}$ may be helpful (at least operationally) for the computation of $f_{k}(C)$ in EST and within this framework one has the following correspondence between loop counting and $f_{k}(C)$ computation:

$$
\begin{aligned}
1 \text { loop } & \Longrightarrow f_{\ln }(C) \ln \lambda+f_{0}(C), \\
2 \text { loops } & \Longrightarrow \lambda^{-2} f_{2}(C) .
\end{aligned}
$$

The main subject of this paper is 2-loop correction $f_{2}(C)$. Technically (but not conceptually) the computation of $f_{2}(C)$ can be well separated from the computation of 1-loop terms $f_{\ln }(C) \ln \lambda+f_{0}(C)$. Therefore in subsequent sections we concentrate on $f_{2}(C)$ whereas a detailed discussion of 1-loop terms is placed to appendices B, C.

In 1-loop approximation (2.12) there appears Laplace determinant which has area, perimeter and cusp divergences (see appendices A.1, A.2). Therefore the completion of the 1-loop computation (i.e. the computation of $f_{\ln }(C), f_{0}(C)$ ) includes two parts:

1) renormalization of Laplace determinant (appendix A.2) using the heat-kernel expansion (appendix A.1),

2) computation of the renormalized Laplace determinant for arbitrary polygons (appendix C) using Schwarz-Christoffel (SC) mapping.

Both parts of this work are well described in literature so that we simply combine all known pieces together in appendices $\mathrm{A}, \mathrm{B}, \mathrm{C}$.

An explicit expression for $f_{\ln }(C)$ can be obtained already at the first step (renormalization of Laplace determinants) - see eq. (B.2). As for $f_{0}(C)$, the situation is more subtle because single Wilson loops $W(C)$ and corresponding functionals $f_{0}(C)$ are not renormalization invariant quantities in MGT. EST provides unambiguous expressions only for renormalization invariant combinations

$$
\sum_{a=1}^{N} m_{a} f_{0}\left(C_{a}\right)
$$

where contours $C_{a}$ and coefficients $m_{a}$ must obey certain balance conditions (B.5), (B.6), (B.8). Once these balance conditions are satisfied, linear combination (2.15) is given by eq. (C.29) derived in appendix $\mathrm{C}$ in terms of parameters of SC mapping.

In the special case when all polygons $C_{a}$ in linear combination $(2.15)$ are triangles one can derive a simpler expression for (2.15) directly in terms of geometric parameters of triangles - see eq. (C.32). 


\section{$2.3 \quad$ 2-loop EST correction}

Using action (2.8), we compute the functional integral of EST in the 2-loop approximation:

$$
\begin{aligned}
W(C) & \rightarrow \int D Y \exp \left\{-\left[\mathcal{S}_{0}+\mathcal{S}_{2}(Y)+\mathcal{S}_{4}(Y)\right]\right\} \\
& \rightarrow \exp \left(-\mathcal{S}_{0}\right) \int D Y\left[1-\mathcal{S}_{4}(Y)\right] \exp \left[-\mathcal{S}_{2}(Y)\right] \\
& =\exp \left(-\mathcal{S}_{0}\right)\left(1-\left\langle\mathcal{S}_{4}\right\rangle\right) \int D Y \exp \left[-\mathcal{S}_{2}(Y)\right]
\end{aligned}
$$

Here

$$
\left\langle\mathcal{S}_{4}\right\rangle=\frac{\int D Y \mathcal{S}_{4}(Y) \exp \left[-\mathcal{S}_{2}(Y)\right]}{\int D Y \exp \left[-\mathcal{S}_{2}(Y)\right]} .
$$

Thus in the 2-loop approximation

$$
\langle W(C)\rangle^{2 \text {-loop }}=\exp \left(-\mathcal{S}_{0}\right)\left[1-\left\langle\mathcal{S}_{4}\right\rangle\right] \int D Y \exp \left[-\mathcal{S}_{2}(Y)\right] .
$$

Comparing this with the 1-loop approximation

$$
\langle W(C)\rangle^{1 \text {-loop }}=\exp \left(-\mathcal{S}_{0}\right) \int D Y \exp \left[-\mathcal{S}_{2}(Y)\right],
$$

we see that

$$
\langle W(C)\rangle^{2 \text {-loop }}=\langle W(C)\rangle^{1 \text {-loop }}\left(1-\left\langle\mathcal{S}_{4}\right\rangle\right),
$$

which leads to

$$
\ln \langle W(C)\rangle^{2 \text {-loop }} \rightarrow \ln \langle W(C)\rangle^{\text {-loop }}-\left\langle\mathcal{S}_{4}\right\rangle .
$$

In fact, one should use the renormalized version of this formula:

$$
\ln \langle W(C)\rangle_{\text {ren }}^{2 \text {-loop }}=\ln \langle W(C)\rangle_{\text {ren }}^{1 \text {-loop }}-\left\langle\mathcal{S}_{4}\right\rangle^{\text {ren }} .
$$

Comparing this with expansion (1.5), we see that

$$
\lambda^{-2} f_{2}(C)=-\left\langle\mathcal{S}_{4}\right\rangle_{\lambda C}^{\mathrm{ren}}
$$

Taking into account eq. (1.9), we obtain

$$
f_{2}(C)=-\left\langle\mathcal{S}_{4}\right\rangle_{C}^{\mathrm{ren}} .
$$

This relation is used in the final step of our computational program (1.20).

Using expression (2.11) for $\mathcal{S}_{4}$, we compute Gaussian integral (2.17)

$$
\begin{aligned}
\frac{1}{\sigma_{0}}\left\langle\mathcal{S}_{4}\right\rangle= & \frac{1}{8} \int_{U(C)} d^{2} x \sum_{\mu, \nu=1}^{2} \sum_{a, b=3}^{D}\left\langle\frac{\partial Y^{a}}{\partial x^{\mu}} \frac{\partial Y^{a}}{\partial x^{\mu}} \frac{\partial Y^{b}}{\partial x^{\nu}} \frac{\partial Y^{b}}{\partial x^{\nu}}\right\rangle \\
& -\frac{1}{4} \int_{U(C)} d^{2} x \sum_{\mu, \nu=1}^{2} \sum_{a, b=3}^{D}\left\langle\frac{\partial Y^{a}}{\partial x^{\mu}} \frac{\partial Y^{b}}{\partial x^{\mu}} \frac{\partial Y^{a}}{\partial x^{\nu}} \frac{\partial Y^{b}}{\partial x^{\nu}}\right\rangle .
\end{aligned}
$$


Now Wick theorem yields a figure-eight-like Feynman diagram

$$
\begin{aligned}
\frac{1}{\sigma_{0}}\left\langle\mathcal{S}_{4}\right\rangle= & \frac{1}{8} \int_{U(C)} d^{2} x \sum_{\mu, \nu=1}^{2} \sum_{a, b=3}^{D}\left[\left\langle\frac{\partial Y^{a}}{\partial x^{\mu}} \frac{\partial Y^{a}}{\partial x^{\mu}}\right\rangle\left\langle\frac{\partial Y^{b}}{\partial x^{\nu}} \frac{\partial Y^{b}}{\partial x^{\nu}}\right\rangle\right. \\
& \left.+2\left\langle\frac{\partial Y^{a}}{\partial x^{\mu}} \frac{\partial Y^{b}}{\partial x^{\nu}}\right\rangle\left\langle\frac{\partial Y^{a}}{\partial x^{\mu}} \frac{\partial Y^{b}}{\partial x^{\nu}}\right\rangle\right] \\
& -\frac{1}{4} \int_{U(C)} d^{2} x \sum_{\mu, \nu=1}^{2} \sum_{a, b=3}^{D}\left[\left\langle\frac{\partial Y^{a}}{\partial x^{\mu}} \frac{\partial Y^{b}}{\partial x^{\mu}}\right\rangle\left\langle\frac{\partial Y^{a}}{\partial x^{\nu}} \frac{\partial Y^{b}}{\partial x^{\nu}}\right\rangle\right. \\
& \left.+\left\langle\frac{\partial Y^{a}}{\partial x^{\mu}} \frac{\partial Y^{a}}{\partial x^{\nu}}\right\rangle\left\langle\frac{\partial Y^{b}}{\partial x^{\mu}} \frac{\partial Y^{b}}{\partial x^{\nu}}\right\rangle+\left\langle\frac{\partial Y^{a}}{\partial x^{\mu}} \frac{\partial Y^{b}}{\partial x^{\nu}}\right\rangle\left\langle\frac{\partial Y^{b}}{\partial x^{\mu}} \frac{\partial Y^{a}}{\partial x^{\nu}}\right\rangle\right],
\end{aligned}
$$

with the propagator

$$
\left\langle\frac{\partial Y^{a}}{\partial x^{\mu}} \frac{\partial Y^{b}}{\partial x^{\nu}}\right\rangle=-\frac{1}{\sigma_{0}} \delta_{a b} G_{\mu \nu}
$$

where

$$
\begin{aligned}
G_{\mu \nu}(x) & =-\left\langle x\left|\partial_{\mu} \frac{1}{\Delta(C)} \partial_{\nu}\right| x\right\rangle, \\
\left\langle y\left|\partial_{\mu} \frac{1}{\Delta(C)} \partial_{\nu}\right| x\right\rangle & =-\frac{\partial}{\partial y^{\mu}} \frac{\partial}{\partial x^{\nu}}\left\langle y\left|\frac{1}{\Delta(C)}\right| x\right\rangle
\end{aligned}
$$

and

$$
\left\langle y\left|\frac{1}{\Delta(C)}\right| x\right\rangle
$$

is Green function of Laplace operator with Dirichlet boundary condition:

$$
\begin{aligned}
\sum_{\mu=1}^{2} \frac{\partial^{2}}{\partial y^{\mu} \partial y^{\mu}}\left\langle y\left|\frac{1}{\Delta(C)}\right| x\right\rangle & =\delta(y-x), \\
\left.\left\langle y\left|\frac{1}{\Delta(C)}\right| x\right\rangle\right|_{y \in C} & =0, \\
\left\langle y\left|\frac{1}{\Delta(C)}\right| x\right\rangle & =\left\langle x\left|\frac{1}{\Delta(C)}\right| y\right\rangle .
\end{aligned}
$$

Now we insert eq. (2.27) into eq. (2.26)

$$
\begin{aligned}
\sigma_{0}\left\langle\mathcal{S}_{4}\right\rangle= & \frac{D-2}{8} \int_{U(C)} d^{2} x \sum_{\mu, \nu=1}^{2}\left[(D-2) G_{\mu \mu} G_{\nu \nu}+2 G_{\mu \nu} G_{\mu \nu}\right] \\
& -\frac{D-2}{4} \int_{U(C)} d^{2} x \sum_{\mu, \nu=1}^{2}\left[G_{\mu \mu} G_{\nu \nu}+(D-1) G_{\mu \nu} G_{\mu \nu}\right] .
\end{aligned}
$$

Thus

$$
\left\langle\mathcal{S}_{4}\right\rangle=-\frac{D-2}{8 \sigma_{0}} \int_{U(C)} d^{2} x \sum_{\mu, \nu=1}^{2}\left[(4-D) G_{\mu \mu} G_{\nu \nu}+2(D-2) G_{\mu \nu} G_{\mu \nu}\right] .
$$


One should keep in mind that the r.h.s. is plagued by divergences. Indeed, nondiagonal matrix element (2.29) has a singularity at $y \rightarrow x$ so that quantity $G_{\mu \nu}(x)$ is divergent for any $x$. This means that on the r.h.s. of eq. (2.35) the integrand is divergent at any point $x$. Nevertheless eq. (2.35) provides a good starting point for the discussion of the renormalization.

\section{Renormalization}

\subsection{Rectangular case: method of K. Dietz and T. Filk}

The naive non-renormalized expression for the 2-loop correction (2.35) allows for arbitrary polygons $C$ and in this sense is common for the current work concentrating on triangles and for refs. [26, 27] devoted to rectangles. But starting from eq. (2.35) the paths of this work and refs. [26, 27] diverge. The main reason is that the authors of refs. [26, 27] use an ultraviolet regularization specific for the rectangular case which cannot be applied to other polygons. The regularization of refs. $[26,27]$ is based on the explicit diagonalization of Laplace operator acting in rectangle

$$
0 \leq x^{\mu} \leq L_{\mu} \quad(\mu=1,2)
$$

with Dirichlet boundary conditions. The spectral problem

$$
-\Delta \psi_{m n}=\lambda_{m n} \psi_{m n}
$$

has the obvious solution

$$
\begin{aligned}
& \lambda_{m n}=\pi^{2}\left(\frac{m^{2}}{L_{1}^{2}}+\frac{n^{2}}{L_{2}^{2}}\right), \\
& \psi_{m n}=\frac{2}{\sqrt{L_{1} L_{2}}} \sin \left(\frac{\pi m x^{1}}{L_{1}}\right) \sin \left(\frac{\pi n x^{2}}{L_{2}}\right) .
\end{aligned}
$$

The method of refs. [26, 27] is based on

1) formal spectral decomposition of $G_{\mu \nu}(2.28)$ in terms of $\lambda_{m n}, \psi_{m n}$ ignoring the divergence of $G_{\mu \nu}$,

2) insertion of this formal expression for $G_{\mu \nu}$ into the r.h.s. of eq. (2.35),

3) formal integration over $x$ in eq. (2.35).

As a result, one obtains an expression for $\left\langle\mathcal{S}_{4}\right\rangle$ in terms of divergent series

$$
\sum_{m_{1} m_{2} \ldots m_{n}} \frac{R_{1}\left(m_{1}, m_{2}, \ldots, m_{n}\right)}{R_{2}\left(m_{1}, m_{2}, \ldots, m_{n}\right)}
$$

where $R_{a}\left(m_{1}, m_{2}, \ldots, m_{n}\right)$ are some polynomials of integer $m_{k}$ (originating from parameters $m, n$ of eigenvalues $\lambda_{m n}$ ). Formal series (3.5) are divergent. Refs. [26, 27] use analytical regularization for numerators $R_{1}$ so that the problem reduces to the computation of sums

$$
\sum_{m_{1} m_{2} \ldots m_{n}} \frac{m_{1}^{\alpha_{1}} m_{2}^{\alpha_{2}} \ldots m_{n}^{\alpha_{n}}}{R_{2}\left(m_{1}, m_{2}, \ldots, m_{n}\right)}
$$


in convergence region of $\alpha_{k}$-space with a subsequent analytical continuation in $\alpha_{k}$ to integer values corresponding to monomials of the original numerator $R_{1}\left(m_{1}, m_{2}, \ldots, m_{n}\right)$ in eq. (3.5).

This approach allows for a complete analytical computation of $f_{2}(C)$ for rectangles $C$ with result (1.16). However, the method is based on the explicit expressions for spectrum (3.3) and for eigenfunctions (3.4) and therefore is limited to the case of rectangles.

From this brief review of Dietz-Filk (DF) method it is clear that DF regularization belongs to the class of last-minute regularizations: one starts from divergent integrals and sums; after a naive formal algebra with these divergent expressions one introduces the regularization in the end of the calculation (the transition from eq. (3.5) to eq. (3.6)). From the rigorous point of view it would be preferable to use some other complete overall regularization of EST from the very beginning, covering all orders of the loop expansion in a consistent universal way (e.g. dimensional regularization). However, in the practical work a compromise between solid theoretical background and computational efficiency is often made in favor of DF method, see. e.g. refs. [18, 19, 24] where DF method was extended to higher orders of the large-size expansion (still as a last-minute regularization). As for tests of the consistency between the theoretically preferable dimensional regularization and the practically efficient DF method, one should mention the case of the correlation function of two parallel Polyakov lines where an explicit calculation of the 2-loop contribution (analog of $f_{2}(C)$ with rectangular contours $C$ ) in dimensional regularization [16] confirmed the original DF result $[26,27]$.

\subsection{General polygons: renormalization based on SC mapping}

As discussed above, the analytical regularization of refs. [26, 27] was designed for rectangles. Our aim is to construct a regularization (and renormalization) procedure for $f_{2}(C)$ with arbitrary polygons $C$. We also want this regularization method to be efficient for practical computations. The first hint for the construction of this regularization procedure comes from the lessons of 1-loop EST corrections. As discussed in appendix C, the explicit analytical computation of 1-loop correction $f_{0}(C)$ is based on SC mapping for polygon $C$. This observation suggests that we should try to construct the regularization and the renormalization for the 2-loop correction $f_{2}(C)$ also in terms of SC mapping.

The next observation is that the naive divergent expression (2.35) has two types of divergences:

1) The integrand is divergent at any point $x$ because $G_{\mu \nu}(x)$ is divergent at any $x$. Therefore first one must renormalize the integrand on the r.h.s. of (2.35).

2) After this step of the renormalization the integrand on the r.h.s. of (2.35) becomes finite in the internal part of the integration region but remains singular on the boundary $C$ so that the integral is still divergent. At this moment we will need the second step of the renormalization.

This two-step renormalization was already announced - see eq. (1.20). Note that in the case of refs. [26, 27] both types of divergences were renormalized using one renormalization procedure based on analytical regularization (3.6) of formal divergent series: discrete 
summation (3.5) of the spectral representation keeps interior and boundary divergences in one pot. But our method based on SC representation requires a separate treatment of interior and boundary divergences.

Thus we have a two-stage renormalization procedure. A detailed description of the two steps will be given below. But already now it makes sense to announce that the second step (renormalization of boundary divergences) will be implemented via a sort of analytical regularization for divergent integrals in SC representation. This SC version of the analytical regularization (applicable to arbitrary polygons $C$ ) is different from the analytical regularization of refs. [26, 27] designed for rectangles and dealing with discrete series.

Similarly to the case of DF method, the analytical regularization based on SC mapping belongs to the class of last-minute regularizations. The main aim is to develop an efficient computational method for the calculation of $f_{2}(C)$ with arbitrary polygonal contours postponing the problem of a rigorous justification for later. The detailed structure of the regularization and renormalization procedure described in this work is limited to the case of the 2-loop contribution $f_{2}(C)$. However, the main ingredient of the method, SC mapping, could be of use also for the computation of higher orders of the large-size expansion with arbitrary polygonal contours.

\subsection{First step of renormalization: from $\left\langle\mathcal{S}_{4}\right\rangle$ to $\left\langle\mathcal{S}_{4}\right\rangle^{\text {ren,1 }}$}

Function $G_{\mu \nu}$ (2.28) is divergent. Indeed, matrix element (2.29) has a singularity in the diagonal limit $y \rightarrow x$ :

$$
\begin{aligned}
\left\langle y\left|\partial_{\mu} \frac{1}{\Delta(C)} \partial_{\nu}\right| x\right\rangle \stackrel{y \rightarrow x}{\sim}-\frac{\partial}{\partial y^{\mu}} \frac{\partial}{\partial x^{\nu}} \frac{1}{2 \pi} \ln |x-y| & =-\frac{r^{2} \delta_{\mu \nu}-2(x-y)_{\mu}(x-y)_{\nu}}{2 \pi r^{4}}, \\
r & =|x-y|
\end{aligned}
$$

This divergence is eliminated by a renormalization of string tension $\sigma_{0}$. Operationally this renormalization corresponds to the replacement of $G_{\mu \nu}(2.28)$ by its finite part

$$
K_{\mu \nu}(x)=\lim _{y \rightarrow x}\left[\left\langle y\left|\partial_{\mu} \frac{1}{-\Delta(C)} \partial_{\nu}\right| x\right\rangle-\frac{r^{2} \delta_{\mu \nu}-2(x-y)_{\mu}(x-y)_{\nu}}{2 \pi r^{4}}\right]
$$

and by the replacement of bare string tension $\sigma_{0}$ with renormalized string tension $\sigma$ appearing in area law (1.1).

After the replacement

$$
\begin{aligned}
G_{\mu \nu} & \rightarrow K_{\mu \nu} \\
\sigma_{0} & \rightarrow \sigma
\end{aligned}
$$

eq. (2.35) takes the form

$$
\left\langle\mathcal{S}_{4}\right\rangle^{\mathrm{ren}, 1}=-\frac{D-2}{8 \sigma} \int_{U(C)} d^{2} x \sum_{\mu, \nu=1}^{2}\left[(4-D) K_{\mu \mu} K_{\nu \nu}+2(D-2) K_{\mu \nu} K_{\mu \nu}\right] .
$$

We use label ren, 1 in $\left\langle\mathcal{S}_{4}\right\rangle^{\text {ren,1 }}$ because the integral on the r.h.s. still contains additional divergences: 
- boundary divergences which appear in $K_{\mu \nu}(x)$ when $x$ approaches boundary $C$ of region $U(C)$,

- extra cusp divergences appearing in polygonal regions $U(C)$ when $x$ approaches a vertex of the polygon.

Thus, the first step $\left\langle\mathcal{S}_{4}\right\rangle \rightarrow\left\langle\mathcal{S}_{4}\right\rangle^{\text {ren,1 }}$ of our renormalization program (1.20) has made the integrand finite in the internal part of $U(C)$ on the r.h.s. of (3.12) but the integral is still divergent because of boundary and cusp singularities of the integrand.

\section{Complex representation}

\subsection{Definitions and conventions}

In order to proceed, it is convenient to pass from Cartesian $2 \mathrm{D}$ coordinates $x$ to complex coordinates $\zeta$. Our conventions for this complexification are

$$
\begin{aligned}
\zeta & =x^{1}+i x^{2}, \\
\zeta^{*} & =x^{1}-i x^{2}, \\
\frac{\partial}{\partial \zeta} & =\frac{1}{2}\left(\frac{\partial}{\partial x^{1}}-i \frac{\partial}{\partial x^{2}}\right), \\
\frac{\partial}{\partial \zeta^{*}} & =\frac{1}{2}\left(\frac{\partial}{\partial x^{1}}+i \frac{\partial}{\partial x^{2}}\right), \\
d^{2} \zeta & =d x^{1} d x^{2}, \\
d s^{2} & =d x^{1} d x^{1}+d x^{2} d x^{2}=d \zeta d \zeta^{*} .
\end{aligned}
$$

We use the metric tensor with components

$$
\begin{aligned}
g_{\zeta \zeta^{*}} & =g_{\zeta^{*} \zeta}=\frac{1}{2}, \\
g_{\zeta \zeta} & =g_{\zeta^{*} \zeta^{*}}=0, \\
g^{\zeta \zeta^{*}} & =g^{\zeta^{*} \zeta}=2, \\
g^{\zeta \zeta} & =g^{\zeta^{*} \zeta^{*}}=0 .
\end{aligned}
$$

For vector $V$

$$
\begin{aligned}
V^{\zeta} & =2 V_{\zeta^{*}}=V^{1}+i V^{2}, \\
V^{\zeta^{*}} & =2 V_{\zeta}=V^{1}-i V^{2} .
\end{aligned}
$$

For symmetric tensor

$$
K_{\mu \nu}=K_{\nu \mu},
$$

we have

$$
\begin{aligned}
K_{\zeta \zeta} & =\frac{1}{4}\left(K_{11}-2 i K_{12}-K_{22}\right), \\
K_{\zeta^{*} \zeta^{*}} & =\frac{1}{4}\left(K_{11}+2 i K_{12}-K_{22}\right), \\
K_{\zeta \zeta^{*}} & =K_{\zeta^{*} \zeta}=\frac{1}{4}\left(K_{11}+K_{22}\right)
\end{aligned}
$$


so that

$$
\begin{aligned}
K_{\mu \nu} K_{\mu \nu} & =8\left(K_{\zeta \zeta^{*}} K_{\zeta \zeta^{*}}+K_{\zeta \zeta} K_{\zeta^{*} \zeta^{*}}\right) \\
K_{\mu \mu} K_{\nu \nu} & =16 K_{\zeta \zeta^{*}} K_{\zeta \zeta^{*}}
\end{aligned}
$$

Now eq. (3.12) becomes

$$
\left\langle\mathcal{S}_{4}\right\rangle^{\mathrm{ren}, 1}=-\frac{2}{\sigma}(D-2) \int_{U(C)} d^{2} x\left[2\left|K_{\zeta \zeta^{*}}\right|^{2}+(D-2)\left|K_{\zeta \zeta}\right|^{2}\right] .
$$

In real and complex representations for the Green function

$$
\left\langle y\left|\frac{1}{\Delta(C)}\right| x\right\rangle,
$$

we assume the equivalence of various notations

$$
|x\rangle=\left|x^{1}, x^{2}\right\rangle=\left|x^{1}+i x^{2}\right\rangle=|\zeta\rangle=\left|\zeta, \zeta^{*}\right\rangle
$$

representing the same coordinate state.

With the above conventions we can rewrite eq. (3.9) in the complex form:

$$
\begin{aligned}
K_{\zeta \zeta}(\zeta) & =\lim _{\zeta^{\prime} \rightarrow \zeta}\left[\frac{\partial}{\partial \zeta^{\prime}} \frac{\partial}{\partial \zeta}\left\langle\zeta^{\prime}\left|\frac{1}{\Delta(C)}\right| \zeta\right\rangle-\frac{1}{4 \pi\left(\zeta^{\prime}-\zeta\right)^{2}}\right], \\
K_{\zeta \zeta^{*}}(\zeta) & =\lim _{\zeta^{\prime} \rightarrow \zeta}\left[\frac{\partial}{\partial \zeta^{\prime}} \frac{\partial}{\partial \zeta^{*}}\left\langle\zeta^{\prime}\left|\frac{1}{\Delta(C)}\right| \zeta\right\rangle\right] .
\end{aligned}
$$

\subsection{Conformal mapping to semiplane}

If one knows a conformal mapping of region $U$ (bounded by Wilson contour $C$ ) to the upper complex semiplane then functions $K_{\zeta \zeta}, K_{\zeta \zeta^{*}}$ can be expressed via this conformal mapping. The computation is done in appendix E. The result is

$$
\begin{aligned}
K_{\zeta \zeta}(\zeta) & =\frac{1}{24 \pi}\{z, \zeta\}, \\
K_{\zeta \zeta^{*}}(\zeta) & =\frac{1}{16 \pi}\left|\frac{\partial z(\zeta)}{\partial \zeta}\right|^{2} \frac{1}{[\operatorname{Im} z(\zeta)]^{2}} .
\end{aligned}
$$

Here $\zeta$ is a complex coordinate in region $U$ and $z(\zeta)$ is the conformal mapping from $U$ to the upper complex $z$-semiplane. We use notation $\{z, \zeta\}$ for Schwarz derivative (D.1).

\subsection{Reduction of $\left\langle\mathcal{S}_{4}\right\rangle^{\text {ren,1 }}$ to basic integrals $I_{1}$ and $I_{2}$}

Inserting expressions (4.24), (4.25) into eq. (4.19), we obtain

$$
\left\langle\mathcal{S}_{4}\right\rangle^{\mathrm{ren}, 1}=-\frac{1}{\sigma} \frac{D-2}{(8 \pi)^{2}}\left[I_{1}+\frac{2}{9}(D-2) I_{2}\right]
$$

where

$$
\begin{aligned}
I_{1} & =\int_{U(C)} d^{2} \zeta\left|\frac{\partial z(\zeta)}{\partial \zeta} \frac{1}{[\operatorname{Im} z(\zeta)]}\right|^{4} \\
I_{2} & =\int_{U(C)} d^{2} \zeta|\{z, \zeta\}|^{2}
\end{aligned}
$$


Next we change the integration variable from $\zeta$ to $z$ in integrals (4.27), (4.28). In expression (4.28) for $I_{2}$ we use property (D.3) of Schwarz derivative

$$
\{z, \zeta\}=-\left(\frac{d z}{d \zeta}\right)^{2}\{\zeta, z\}
$$

Thus

$$
\begin{aligned}
& I_{1}=\int_{\mathbb{C}_{+}} d^{2} z\left|\frac{d \zeta}{d z}\right|^{-2}|\operatorname{Im} z|^{-4}, \\
& I_{2}=\int_{\mathbb{C}_{+}} d^{2} z\left|\frac{d \zeta}{d z}\right|^{-2}|\{\zeta, z\}|^{2} .
\end{aligned}
$$

Here the integration runs over upper complex semiplane

$$
\mathbb{C}_{+}=\{z: \operatorname{Im} z>0\} .
$$

\section{$5 \quad$ Polygons and SC mapping}

\subsection{SC mapping with finite vertices}

In the case of polygons, conformal mapping $\zeta(z)$ is known as SC mapping. SC mapping $\zeta(z)$ for a polygon with $n_{\mathrm{v}}$ vertices is defined by differential equation

$$
\frac{d \zeta}{d z}=A \prod_{k=1}^{n_{\mathrm{v}}}\left(z-z_{k}\right)^{-\beta_{k}}
$$

Parameters $\beta_{k}$ determine interior angles of the polygon:

$$
\theta_{k}=\pi\left(1-\beta_{k}\right)
$$

Angles $\theta_{k}$ satisfy geometric conditions

$$
\begin{gathered}
0<\theta_{k}<2 \pi, \theta_{k} \neq \pi, \\
\sum_{k=1}^{n_{\mathrm{v}}}\left(\pi-\theta_{k}\right)=2 \pi
\end{gathered}
$$

so that parameters $\beta_{k}$ obey constraints

$$
\begin{aligned}
& -1<\beta_{k}<1, \beta_{k} \neq 0, \\
& \sum_{k=1}^{n_{\mathrm{v}}} \beta_{k}=2 .
\end{aligned}
$$

Sometimes it is convenient to use parameters

$$
\alpha_{k}=1-\beta_{k}=\frac{\theta_{k}}{\pi}
$$


For SC mapping (5.1), Schwarz derivative $\{\zeta, z\}$ (D.1) can be easily computed:

$$
\{\zeta, z\}=\left[\sum_{k=1}^{n_{\mathrm{v}}} \frac{\beta_{k}}{\left(z-z_{k}\right)^{2}}\right]-\frac{1}{2}\left(\sum_{k=1}^{n_{\mathrm{v}}} \frac{\beta_{k}}{z-z_{k}}\right)^{2} .
$$

Now eqs. (4.30), (4.31) lead to

$$
\begin{aligned}
& I_{1}=|A|^{-2} \int_{\mathbb{C}_{+}} d^{2} z \prod_{k=1}^{n_{\mathrm{v}}}\left|z-z_{k}\right|^{2 \beta_{k}}|\operatorname{Im} z|^{-4} \\
& I_{2}=|A|^{-2} \int_{\mathbb{C}_{+}} d^{2} z \prod_{k=1}^{n_{\mathrm{v}}}\left|z-z_{k}\right|^{2 \beta_{k}}\left|\left[\sum_{k=1}^{n_{\mathrm{v}}} \frac{\beta_{k}}{\left(z-z_{k}\right)^{2}}\right]-\frac{1}{2}\left(\sum_{k=1}^{n_{\mathrm{v}}} \frac{\beta_{k}}{z-z_{k}}\right)^{2}\right|^{2} .
\end{aligned}
$$

Thus

$$
I_{a}=|A|^{-2} \int_{\mathbb{C}_{+}} d^{2} z \prod_{k=1}^{n_{\mathrm{v}}}\left|z-z_{k}\right|^{2 \beta_{k}} Q_{a}\left(z, z^{*}\right)
$$

where

$$
\begin{aligned}
Q_{1}\left(z, z^{*}\right) & =|\operatorname{Im} z|^{-4}, \\
Q_{2}\left(z, z^{*}\right) & =\left|\left[\sum_{k=1}^{n_{\mathrm{v}}} \frac{\beta_{k}}{\left(z-z_{k}\right)^{2}}\right]-\frac{1}{2}\left(\sum_{k=1}^{n_{\mathrm{V}}} \frac{\beta_{k}}{z-z_{k}}\right)^{2}\right|^{2} .
\end{aligned}
$$

\section{$5.2 \quad \mathrm{SL}(2, \mathbb{R})$ symmetry}

It is well-known that linear fractional transformations

$$
z^{\prime}=\frac{a z+b}{c z+d}
$$

with real coefficients

$$
\begin{aligned}
& a, b, c, d \in \mathbb{R} \\
& a d-b c=1
\end{aligned}
$$

map the upper complex semiplane to itself. The group of these transformations essentially coincides with $\mathrm{SL}(2, \mathbb{R})$ group of matrices

$$
\left(\begin{array}{ll}
a & b \\
c & d
\end{array}\right)
$$

with unit determinant (more exactly, with $\mathrm{SL}(2, \mathbb{R}) / \mathbb{Z}_{2}$ ).

For a given polygon its SC mapping is defined up to the freedom of these linear fractional transformations. In particular, this allows for the freedom of changing $\mathrm{SC}$ vertices $z_{k}$

$$
z_{k}^{\prime}=\frac{a z_{k}+b}{c z_{k}+d}
$$

Naive expressions (5.12), (5.13) are formally invariant under this symmetry. But when it comes to the renormalization of ultraviolet divergences in naive integrals, this $\operatorname{SL}(2, \mathbb{R})$ symmetry imposes nontrivial constraints on the renormalization procedure. 


\subsection{SC mapping with one vertex at infinity}

The freedom of linear fractional transformations (5.18) allows for taking one SC vertex to infinity. Let us put vertex $z_{n_{\mathrm{v}}}$ to infinity:

$$
\left|z_{n_{\mathrm{v}}}\right| \rightarrow \infty
$$

When taking this limit, one should tune parameter $A=A\left(z_{n_{\mathrm{v}}}\right)$ in eq. (5.1) so that

$$
\lim _{\left|z_{n_{\mathrm{v}}}\right| \rightarrow \infty} A\left(z_{n_{\mathrm{v}}}\right)\left(z-z_{n_{\mathrm{v}}}\right)^{-\beta_{n_{\mathrm{v}}}}=\lim _{\left|z_{n_{\mathrm{v}}}\right| \rightarrow \infty} A\left(z_{n_{\mathrm{v}}}\right)\left(-z_{n_{\mathrm{v}}}\right)^{-\beta_{n_{\mathrm{v}}}}=\tilde{A}=\text { finite } \neq 0
$$

Then SC equation (5.1) reduces to

$$
\frac{d \zeta}{d z}=\tilde{A} \prod_{k=1}^{n_{\mathrm{v}}-1}\left(z-z_{k}\right)^{-\beta_{k}}
$$

This equation does not contain parameter $\beta_{n_{\mathrm{v}}}$ but it still can be defined by eq. (5.6)

$$
\beta_{n_{\mathrm{v}}}=2-\sum_{k=1}^{n_{\mathrm{v}}-1} \beta_{k}
$$

and obeys condition (5.5) together with other parameters $\beta_{k}$ :

$$
-1<\beta_{k}<1 \quad\left(1 \leq k \leq n_{\mathrm{v}}\right) .
$$

The expression for Schwarz derivative (5.8) of mapping (5.21) can be obtained by taking limit (5.19) in eq. (5.8):

$$
\{\zeta, z\}=\left[\sum_{k=1}^{n_{\mathrm{v}}-1} \frac{\beta_{k}}{\left(z-z_{k}\right)^{2}}\right]-\frac{1}{2}\left(\sum_{k=1}^{n_{\mathrm{v}}-1} \frac{\beta_{k}}{z-z_{k}}\right)^{2} .
$$

Now we apply general formulas (5.11)-(5.12) to SC mapping (5.21):

$$
I_{a}=|\tilde{A}|^{-2} \int_{\mathbb{C}_{+}} d^{2} z \prod_{k=1}^{n_{\mathrm{v}}-1}\left|z-z_{k}\right|^{2 \beta_{k}} \tilde{Q}_{a}\left(z, z^{*}\right) .
$$

Here

$$
\begin{aligned}
& \tilde{Q}_{1}\left(z, z^{*}\right)=|\operatorname{Im} z|^{-4}, \\
& \tilde{Q}_{2}\left(z, z^{*}\right)=\left|\left[\sum_{k=1}^{n_{\mathrm{v}}-1} \frac{\beta_{k}}{\left(z-z_{k}\right)^{2}}\right]-\frac{1}{2}\left(\sum_{k=1}^{n_{\mathrm{v}}-1} \frac{\beta_{k}}{z-z_{k}}\right)^{2}\right|^{2} .
\end{aligned}
$$




\subsection{SC vertex at infinity: pro and contra}

Thus we have two versions of SC representation for $I_{a}$. Representation (5.11) is based on SC mapping with finite vertices $z_{k}$ whereas representation (5.25) assumes that SC vertex $z_{n_{\mathrm{v}}}$ is taken to infinity. Both integrals (5.11), (5.25) are divergent. These integrals must be renormalized (by the second step $\left\langle\mathcal{S}_{4}\right\rangle^{\text {ren,1 }} \rightarrow\left\langle\mathcal{S}_{4}\right\rangle^{\text {ren }}$ of our renormalization program (1.20)) and our plan is to use analytical regularization. In principle, our procedure of analytical regularization can be formulated for both versions of SC mapping with the same final renormalized result for $I_{a}^{\text {ren }}$. But when it comes to the practical work, the SC representation with one vertex at infinity seems to be preferable. One advantage of this approach is obvious: keeping one SC vertex at infinity we significantly simplify all integrals. The second argument against SC mapping with finite vertices is that parameters $\beta_{k}$ must obey constraint (5.6). This constraint is critical at some steps of analytical regularization so that one meets the problem of analytical continuation in $\beta_{k}$ under constraint (5.6) which leads to some technical (solvable but annoying) problems.

As was discussed in section 5.2, in the case of SC mapping with all SC vertices being finite we have a powerful constraint of $\operatorname{SL}(2, \mathbb{R})$ symmetry on allowed renormalization procedures. Our choice to construct renormalization in terms of SC mapping with one vertex at infinity makes $\mathrm{SL}(2, \mathbb{R})$ symmetry rather implicit. We are left with the freedom of choice: which vertex of the polygon is associated with the SC vertex taken to infinity. The result of the renormalization must be independent of this choice.

\section{Analytical regularization}

\subsection{Functions $M_{P}^{(n)}$}

Divergent integrals (5.25) representing $I_{1}, I_{2}$ belong to the class of integrals

$$
M_{P}^{(n) \text { nonren }}\left(\left\{\gamma_{k}\right\}_{k=0}^{n},\left\{z_{k}\right\}_{k=1}^{n}\right)=\int_{\mathbb{C}_{+}} d^{2} z|\operatorname{Im} z|^{\gamma_{0}} \prod_{k=1}^{n}\left|z-z_{k}\right|^{\gamma_{k}} P\left(z, z^{*}\right)
$$

where

$$
n \geq 2 \text {, }
$$

$P\left(z, z^{*}\right)$ is a polynomial and $z_{k}(1 \leq k \leq n)$ are different real numbers:

$$
z_{j} \neq z_{k} \text { if } j \neq k \text {. }
$$

We use label nonren in $M_{P}^{(n) \text { nonren }}$ because the integral on the r.h.s. of eq. (6.1) may be divergent.

Expression (5.25) can be rewritten for $I_{1}$ in the form

$$
\begin{aligned}
I_{1} & =|\tilde{A}|^{-2} \int_{\mathbb{C}_{+}} d^{2} z \prod_{k=1}^{n_{\mathrm{v}}-1}\left|z-z_{k}\right|^{2 \beta_{k}}|\operatorname{Im} z|^{-4} \\
& =M_{1}^{\left(n_{\mathrm{v}}-1\right) \text { nonren }}\left(-4,\left\{2 \beta_{k}\right\}_{k=1}^{n_{\mathrm{v}}-1},\left\{z_{k}\right\}_{k=1}^{n_{\mathrm{v}}-1}\right)
\end{aligned}
$$

where $M_{1}^{(n) \text { nonren }}$ is $M_{P}^{(n) \text { nonren }}$ with trivial constant polynomial $P\left(z, z^{*}\right) \equiv 1$. 
For $I_{2}$ we find from eqs. (5.25) and (5.27)

$$
\begin{aligned}
I_{2} & =|\tilde{A}|^{-2} \int_{\mathbb{C}_{+}} d^{2} z \prod_{k=1}^{n_{\mathrm{v}}-1}\left|z-z_{k}\right|^{2 \beta_{k}}\left|\left[\sum_{k=1}^{n_{\mathrm{v}}-1} \frac{\beta_{k}}{\left(z-z_{k}\right)^{2}}\right]-\frac{1}{2}\left(\sum_{k=1}^{n_{\mathrm{v}}-1} \frac{\beta_{k}}{z-z_{k}}\right)^{2}\right|^{2} \\
& =|\tilde{A}|^{-2} \int_{\mathbb{C}_{+}} d^{2} z \prod_{k=1}^{n_{\mathrm{v}}-1}\left|z-z_{k}\right|^{2 \beta_{k}-4} P_{2}\left(z, z^{*}\right) \\
& =M_{P_{2}}^{\left(n_{\mathrm{v}}-1\right) \text { nonren }}\left(0,\left\{2 \beta_{k}-4\right\}_{k=1}^{n_{\mathrm{v}}-1},\left\{z_{k}\right\}_{k=1}^{n_{\mathrm{v}}-1}\right)
\end{aligned}
$$

where polynomial

$$
P_{2}\left(z, z^{*}\right)=T_{2}(z)\left[T_{2}(z)\right]^{*}
$$

is defined via holomorphic polynomial

$$
T_{2}(z)=\left\{\left[\sum_{k=1}^{n_{\mathrm{v}}-1} \frac{\beta_{k}}{\left(z-z_{k}\right)^{2}}\right]-\frac{1}{2}\left(\sum_{k=1}^{n_{\mathrm{v}}-1} \frac{\beta_{k}}{z-z_{k}}\right)^{2}\right\} \prod_{k=1}^{n_{\mathrm{v}}-1}\left(z-z_{k}\right)^{2} .
$$

One can construct the analytical regularization and renormalization of integrals (6.1) by analogy with the theory of Euler B-function

$$
B\left(\gamma_{1}, \gamma_{2}\right)=\int_{0}^{1} d x x^{\gamma_{1}-1}(1-x)^{\gamma_{2}-1}
$$

First we define functions $M_{P}^{(n)}\left(\left\{\gamma_{k}\right\}_{k=0}^{n},\left\{z_{k}\right\}_{k=1}^{n}\right)$ by integrals (6.1) in the region of parameters $\left\{\gamma_{k}\right\}_{k=0}^{n}$ where these integrals are convergent and then perform analytical continuation in variables $\left\{\gamma_{k}\right\}_{k=0}^{n}$ to physical values appearing in eqs. (6.4), (6.5). This analytical continuation will be denoted as

$$
\begin{aligned}
& M_{P}^{(n)}\left(\left\{\gamma_{k}\right\}_{k=0}^{n},\left\{z_{k}\right\}_{k=1}^{n}\right) \\
& =\text { analyt. cont. in } \gamma_{k}\left[\int_{\mathbb{C}_{+}} d^{2} z|\operatorname{Im} z|^{\gamma_{0}} \prod_{k=1}^{n}\left|z-z_{k}\right|^{\gamma_{k}} P\left(z, z^{*}\right)\right] .
\end{aligned}
$$

The details of the analytical continuation are discussed in section 6.2.

Once analytical function $M_{P}^{(n)}\left(\left\{\gamma_{k}\right\}_{k=0}^{n},\left\{z_{k}\right\}_{k=1}^{n}\right)$ is constructed, one can use it to define analytical renormalization of $I_{1}$ and $I_{2}$ by replacing $M_{P}^{(n) \text { nonren }} \rightarrow M_{P}^{(n)}$ in eqs. (6.4), (6.5)

$$
\begin{aligned}
& I_{1}^{\mathrm{ren}}=|\tilde{A}|^{-2} M_{1}^{\left(n_{\mathrm{v}}-1\right)}\left(-4,\left\{2 \beta_{k}\right\}_{k=1}^{n_{\mathrm{v}}-1},\left\{z_{k}\right\}_{k=1}^{n_{\mathrm{v}}-1}\right), \\
& I_{2}^{\mathrm{ren}}=|\tilde{A}|^{-2} M_{P_{2}}^{\left(n_{\mathrm{v}}-1\right)}\left(0,\left\{2 \beta_{k}-4\right\}_{k=1}^{n_{\mathrm{v}}-1},\left\{z_{k}\right\}_{k=1}^{n_{\mathrm{v}}-1}\right) .
\end{aligned}
$$

These values of $I_{1}^{\text {ren }}, I_{2}^{\text {ren }}$ must be used in the completely renormalized version of eq. (4.26)

$$
\left\langle\mathcal{S}_{4}\right\rangle^{\mathrm{ren}}=-\frac{1}{\sigma} \frac{D-2}{(8 \pi)^{2}}\left[I_{1}^{\mathrm{ren}}+\frac{2}{9}(D-2) I_{2}^{\mathrm{ren}}\right] .
$$

The transition to eqs. (6.10), (6.11), (6.12) corresponds to the second step $\left\langle\mathcal{S}_{4}\right\rangle^{\mathrm{ren}, 1} \rightarrow\left\langle\mathcal{S}_{4}\right\rangle^{\mathrm{ren}}$ of our renormalization program (1.20). 


\subsection{From divergent integrals to meromorphic functions}

The suggested scheme of analytical renormalization raises many questions if one wants a mathematically impeccable implementation of this program. On the other hand, in practical calculations one can typically (but not always) perform formal mathematical operations with divergent integrals without wasting time for a careful justification of these naive formal manipulations. A careful mathematical theory of functions $M_{P}^{(n)}$ for arbitrary $n$ and arbitrary polynomials $P$ can be constructed. But in the framework of the current work devoted to triangular Wilson loops we need functions $M_{P}^{(n)}$ only for the special case $n=2$ and for rather simple polynomials $P$. In this simple case the integrals can be computed explicitly and the problem of analytical continuation can be solved by using these explicit expressions rather than invoking the general theory of functions $M_{P}^{(n)}$ with arbitrary $n$ and arbitrary $P$.

Therefore the complete description of the theory of functions $M_{P}^{(n)}$ will be presented in a separate work devoted to Wilson loops with arbitrary polygonal contours. Here only a list of main results will be given without proofs and derivations.

When one starts with the construction of the rigorous theory of functions $M_{P}^{(n)}$ one meets several questions:

1) Is the region of parameters $\left\{\gamma_{k}\right\}_{k=0}^{n}$ where integral (6.9) is convergent non-empty? In other words, do we have a starting region for the construction of the analytical continuation?

2) Does the analytical continuation depend on the path?

3 ) Is the result of the analytical continuation regular at final physical points appearing on the r.h.s. of eqs. (6.10), (6.11)?

The brief answers are:

1) The region of parameters $\left\{\gamma_{k}\right\}_{k=0}^{n}$ where integral (6.9) is convergent is non-empty for any fixed $\left\{z_{k}\right\}_{k=1}^{n}$ and for any fixed $P$.

2) Starting from function $M_{P}^{(n)}\left(\left\{\gamma_{k}\right\}_{k=0}^{n},\left\{z_{k}\right\}_{k=1}^{n}\right)$ defined by integral (6.9) in the convergence region and performing analytical continuation in $\mathbb{C}^{n+1}$ space of parameters $\left\{\gamma_{k}\right\}_{k=0}^{n}$ (at fixed $\left\{z_{k}\right\}_{k=1}^{n}$ and fixed $P$ ), one arrives at meromorphic function $M_{P}^{(n)}$ of $\left\{\gamma_{k}\right\}_{k=0}^{n}$ (in the sense of theory of several complex variables). In practical terms this means that analytical continuation of $M_{P}^{(n)}$ is independent of the path in the multidimensional space of complex $\left\{\gamma_{k}\right\}_{k=0}^{n}$ but one can meet pole singularities.

3) One can show that $\left\{\gamma_{k}\right\}_{k=0}^{n}$ arguments of $M_{P}^{(n)}$ appearing on the r.h.s. of eqs. (6.10), (6.11) are regular points of meromorphic function $M_{P}^{(n)}$ (if parameters $\beta_{k}$ satisfy geometric constraint (5.23)). In other words, expressions $(6.10),(6.11)$ give finite and unambiguous expressions for $I_{1}^{\text {ren }}$ and $I_{2}^{\text {ren }}$.

A careful proof of these statements for arbitrary $n \geq 2$ (i.e. for polygons with arbitrary number of vertices $n_{\mathrm{v}} \geq 3$ ) requires some effort. We postpone the proof till a separate work 
devoted to arbitrary polygons. In the case of triangles, i.e. for $n=n_{\mathrm{v}}-1=2$ many of the above general properties can be seen directly from explicit expressions computed in appendices $\mathrm{G}, \mathrm{H}$.

\subsection{Functions $\Pi_{P}^{(n)}$}

The above discussion of the analytical regularization proceeded in terms of functions $M_{P}^{(n)}$. These functions are convenient for the preliminary description of our analytical regularization. But when it comes to the real work (including proofs of the announced statements and the practical calculation of $f_{2}(C)$ for various polygons) then it makes sense to use other basic functions which differ from $M_{P}^{(n)}\left(\left\{\gamma_{k}\right\}_{k=0}^{n},\left\{z_{k}\right\}_{k=1}^{n}\right)$ by a simple linear change of arguments. The new functions are defined by the relation

$$
\Pi_{P}^{(n)}\left(\alpha,\left\{\gamma_{k}\right\}_{k=1}^{n},\left\{z_{k}\right\}_{k=1}^{n}\right)=M_{P}^{(n)}\left(\alpha-1,\left\{\gamma_{k}-\alpha-1\right\}_{k=1}^{n},\left\{z_{k}\right\}_{k=1}^{n}\right)
$$

with the inverse expression

$$
M_{P}^{(n)}\left(\gamma_{0},\left\{\gamma_{k}\right\}_{k=1}^{n},\left\{z_{k}\right\}_{k=1}^{n}\right)=\Pi_{P}^{(n)}\left(\gamma_{0}+1,\left\{\gamma_{k}+\gamma_{0}+2\right\}_{k=1}^{n},\left\{z_{k}\right\}_{k=1}^{n}\right) .
$$

Using eq. (6.9), we find that functions $\Pi_{P}^{(n)}$ are given by analytical continuation in $\mathbb{C}^{n+1}$ space of parameters $\alpha,\left\{\gamma_{k}\right\}_{k=1}^{n}$

$$
\begin{aligned}
& \Pi_{P}^{(n)}\left(\alpha,\left\{\gamma_{k}\right\}_{k=1}^{n},\left\{z_{k}\right\}_{k=1}^{n}\right) \\
& =\text { analyt. cont. in } \alpha, \gamma_{k}\left[\int_{\mathbb{C}_{+}} d^{2} z|\operatorname{Im} z|^{\alpha-1} \prod_{k=1}^{n}\left|z-z_{k}\right|^{\gamma_{k}-\alpha-1} P\left(z, z^{*}\right)\right] .
\end{aligned}
$$

In terms of functions $\Pi_{P}^{(n)}$ relations $(6.10),(6.11)$ take the form

$$
\begin{aligned}
& I_{1}^{\mathrm{ren}}=|\tilde{A}|^{-2} \Pi_{1}^{\left(n_{\mathrm{v}}-1\right)}\left(-3,\left\{2 \beta_{k}-2\right\}_{k=1}^{n_{\mathrm{v}}-1},\left\{z_{k}\right\}_{k=1}^{n_{\mathrm{v}}-1}\right), \\
& I_{2}^{\mathrm{ren}}=|\tilde{A}|^{-2} \Pi_{P_{2}}^{\left(n_{\mathrm{v}}-1\right)}\left(1,\left\{2 \beta_{k}-2\right\}_{k=1}^{n_{\mathrm{v}}-1},\left\{z_{k}\right\}_{k=1}^{n_{\mathrm{v}}-1}\right) .
\end{aligned}
$$

Functions $\Pi_{P}^{(n)}$ inherit properties of functions $M_{P}^{(n)}$ discussed in section 6.2. In particular, functions $\Pi_{P}^{(n)}\left(\alpha,\left\{\gamma_{k}\right\}_{k=1}^{n},\left\{z_{k}\right\}_{k=1}^{n}\right)$ are meromorphic functions in the $\mathbb{C}^{n+1}$ space of complex parameters $\alpha,\left\{\gamma_{k}\right\}_{k=1}^{n}$ and are regular at values appearing on the r.h.s. of eqs. (6.16), (6.17) if geometric condition (5.23) holds.

\section{Calculation of $f_{2}(C)$ for triangles}

\subsection{Case of triangles}

In the case of triangles eqs. (6.16), (6.17) take the form

$$
\begin{aligned}
& I_{1}^{\text {ren }}=|\tilde{A}|^{-2} \Pi_{1}^{(2)}\left(-3,\left\{2 \beta_{1}-2,2 \beta_{2}-2\right\},\left\{z_{1}, z_{2}\right\}\right), \\
& I_{2}^{\text {ren }}=|\tilde{A}|^{-2} \Pi_{P_{2}}^{(2)}\left(1,\left\{2 \beta_{1}-2,2 \beta_{2}-2\right\},\left\{z_{1}, z_{2}\right\}\right) .
\end{aligned}
$$


Below we will see that function $\Pi_{1}^{(2)}\left(\alpha,\left\{\gamma_{1}, \gamma_{2}\right\},\left\{z_{1}, z_{2}\right\}\right)$ with arbitrary arguments can be easily computed analytically, which will immediately lead to the result for $I_{1}^{\text {ren }}$. As for $I_{2}^{\text {ren }}$, one can compute $\Pi_{P}^{(2)}\left(\alpha,\left\{\gamma_{1}, \gamma_{2}\right\},\left\{z_{1}, z_{2}\right\}\right)$ on the subspace $\alpha=1$ with arbitrary $\gamma_{1}, \gamma_{2}$, which is sufficient for the computation of $I_{2}^{\text {ren }}(7.2)$.

Without losing generality we perform the computation of $I_{1}^{\text {ren }}$ and $I_{2}^{\text {ren }}$ choosing $\mathrm{SC}$ vertices

$$
z_{1}=0, \quad z_{2}=1
$$

\subsection{Calculation of $I_{1}^{\text {ren }}$}

In appendix $G$ we derive expression (G.10) for function $\Pi_{1}^{(2)}$. Using this result in our expression (7.1) for $I_{1}^{\text {ren }}$, we find

$$
I_{1}^{\mathrm{ren}}=|\tilde{A}|^{-2} \frac{2 \pi}{3} \frac{\Gamma\left(\beta_{1}-1\right)}{\Gamma\left(-\beta_{1}\right)} \frac{\Gamma\left(\beta_{2}-1\right)}{\Gamma\left(-\beta_{2}\right)} \frac{\Gamma\left(1-\beta_{1}-\beta_{2}\right)}{\Gamma\left(\beta_{1}+\beta_{2}-2\right)} .
$$

Next we express parameter $|\tilde{A}|$ via area $S(C)$ of the triangle using eq. (F.11) and simplify the result using expression (F.6) for $\beta_{3}$ :

$$
\begin{aligned}
I_{1}^{\text {ren }}= & \frac{\pi^{2}}{3 S(C)}\left[\frac{\Gamma\left(1-\beta_{1}\right) \Gamma\left(1-\beta_{2}\right) \Gamma\left(1-\beta_{3}\right)}{\Gamma\left(\beta_{1}\right) \Gamma\left(\beta_{2}\right) \Gamma\left(\beta_{3}\right)}\right] \\
& \times\left[\frac{\Gamma\left(\beta_{1}-1\right)}{\Gamma\left(-\beta_{1}\right)} \frac{\Gamma\left(\beta_{2}-1\right)}{\Gamma\left(-\beta_{2}\right)} \frac{\Gamma\left(\beta_{3}-1\right)}{\Gamma\left(-\beta_{3}\right)}\right] \\
= & \frac{\pi^{2}}{3 S(C)} \frac{\beta_{1}}{1-\beta_{1}} \frac{\beta_{2}}{1-\beta_{2}} \frac{\beta_{3}}{1-\beta_{3}} .
\end{aligned}
$$

Thus

$$
I_{1}^{\mathrm{ren}}=\frac{\pi^{2}}{3 S(C)} \prod_{k=1}^{3} \frac{\beta_{k}}{1-\beta_{k}} .
$$

\subsection{Calculation of $I_{2}^{\text {ren }}$}

Now we turn to the computation of $I_{2}^{\text {ren }}$, using its expression (7.2) via $\Pi_{P_{2}}^{(2)}$. Remember that polynomial $P_{2}$ can be expressed via polynomial $T_{2}$ according to eq. (6.6). Let us compute polynomial $T_{2}(z)$ (6.6) for triangles $\left(n_{\mathrm{v}}=3\right)$ with $z_{k}$ given by eq. (7.3). We have

$$
\begin{aligned}
T_{2}(z)= & \left\{\left[\sum_{k=1}^{2} \frac{\beta_{k}}{\left(z-z_{k}\right)^{2}}\right]-\frac{1}{2}\left(\sum_{k=1}^{2} \frac{\beta_{k}}{z-z_{k}}\right)^{2}\right\} \prod_{k=1}^{2}\left(z-z_{k}\right)^{2} \\
= & \left\{\frac{\beta_{1}}{z^{2}}+\frac{\beta_{2}}{(z-1)^{2}}-\frac{1}{2}\left(\frac{\beta_{1}}{z}+\frac{\beta_{2}}{z-1}\right)^{2}\right\} z^{2}(z-1)^{2} \\
= & {\left[\beta_{1}\left(1-\frac{1}{2} \beta_{1}\right)+\beta_{2}\left(1-\frac{1}{2} \beta_{2}\right)-\beta_{1} \beta_{2}\right] z^{2} } \\
& +\left[-2 \beta_{1}+\beta_{1}\left(\beta_{1}+\beta_{2}\right)\right] z+\beta_{1}\left(1-\frac{1}{2} \beta_{1}\right) .
\end{aligned}
$$


This can be rewritten as

$$
T_{2}(z)=\sum_{n=0}^{2} d_{n} z^{n}
$$

where

$$
\begin{aligned}
& d_{0}=\beta_{1}\left(1-\frac{1}{2} \beta_{1}\right), \\
& d_{1}=-2 \beta_{1}+\beta_{1}\left(\beta_{1}+\beta_{2}\right), \\
& d_{2}=\left[\beta_{1}\left(1-\frac{1}{2} \beta_{1}\right)+\beta_{2}\left(1-\frac{1}{2} \beta_{2}\right)-\beta_{1} \beta_{2}\right] .
\end{aligned}
$$

Now eq. (6.6) takes the form

$$
P_{2}\left(z, z^{*}\right)=T_{2}(z)\left[T_{2}(z)\right]^{*}=\left(\sum_{m=0}^{2} d_{m} z^{m}\right)\left(\sum_{n=0}^{2} d_{n} z^{* n}\right) .
$$

Applying expression (H.13) for $\Pi_{P}^{(2)}\left(1,\left\{\gamma_{1}, \gamma_{2}\right\},\{0,1\}\right)$ derived in appendix $\mathrm{H}$ to our case, we find

$$
\begin{aligned}
\Pi_{P_{2}}^{(2)}\left(1,\left\{\gamma_{1}, \gamma_{2}\right\},\{0,1\}\right)= & \frac{\pi}{2} \frac{\sin \left(\pi \frac{\gamma_{1}}{2}\right)}{\sin \left(\pi \frac{\gamma_{1}+\gamma_{2}}{2}\right)} \frac{\Gamma\left(\frac{\gamma_{2}}{2}\right)}{\Gamma\left(1-\frac{\gamma_{2}}{2}\right)} \\
& \times\left[\sum_{m} d_{m} \frac{\Gamma\left(\frac{\gamma_{1}}{2}+m\right)}{\Gamma\left(\frac{\gamma_{1}+\gamma_{2}}{2}+m\right)}\right]^{2} .
\end{aligned}
$$

In eq. (6.17) we are interested in the case

$$
\gamma_{k}=2 \beta_{k}-2
$$

where eq. (7.13) gives

$$
\begin{aligned}
\Pi_{P_{2}}^{(2)}\left(1,\left\{2 \beta_{1}-2,2 \beta_{2}-2\right\},\{0,1\}\right)= & -\frac{\pi}{2} \frac{\sin \left(\pi \beta_{1}\right)}{\sin \left(\pi\left(\beta_{1}+\beta_{2}\right)\right)} \frac{\Gamma\left(\beta_{2}-1\right)}{\Gamma\left(2-\beta_{2}\right)} \\
& \times\left[\sum_{m} d_{m} \frac{\Gamma\left(\beta_{1}-1+m\right)}{\Gamma\left(\beta_{1}+\beta_{2}-2+m\right)}\right]^{2} .
\end{aligned}
$$

Using eqs. (7.9), (7.10), (7.11), we compute

$$
\sum_{m=0}^{2} d_{m} \frac{\Gamma\left(\beta_{1}+m\right)}{\Gamma\left(\beta_{1}+\beta_{2}+m\right)}=\frac{1}{2} \frac{\Gamma\left(\beta_{1}-1\right)}{\Gamma\left(\beta_{1}+\beta_{2}\right)} \beta_{1} \beta_{2}\left(\beta_{1}+\beta_{2}-2\right)
$$

and insert this into eq. (7.15)

$$
\begin{aligned}
& \Pi_{P_{2}}^{(2)}\left(1,\left\{2 \beta_{1}-2,2 \beta_{2}-2\right\},\{0,1\}\right) \\
& =-\frac{\pi}{8} \frac{\sin \left(\pi \beta_{1}\right)}{\sin \left(\pi\left(\beta_{1}+\beta_{2}\right)\right)} \frac{\Gamma\left(\beta_{2}-1\right)}{\Gamma\left(2-\beta_{2}\right)}\left[\frac{\Gamma\left(\beta_{1}-1\right)}{\Gamma\left(\beta_{1}+\beta_{2}\right)} \beta_{1} \beta_{2}\left(\beta_{1}+\beta_{2}-2\right)\right]^{2} .
\end{aligned}
$$


We introduce according to eq. (5.22)

$$
\beta_{3}=2-\beta_{1}-\beta_{2}
$$

and simplify eq. (7.17)

$$
\Pi_{P_{2}}^{(2)}\left(1,\left\{2 \beta_{1}-2,2 \beta_{2}-2\right\},\{0,1\}\right)=\frac{\pi}{8} \prod_{k=1}^{3}\left[\frac{\beta_{k}^{2} \Gamma\left(\beta_{k}-1\right)}{\Gamma\left(2-\beta_{k}\right)}\right] .
$$

We insert this into eq. (6.17)

$$
I_{2}^{\mathrm{ren}}=|\tilde{A}|^{-2} \frac{\pi}{8} \prod_{k=1}^{3}\left[\frac{\beta_{k}^{2} \Gamma\left(\beta_{k}-1\right)}{\Gamma\left(2-\beta_{k}\right)}\right] .
$$

Now we insert $|\tilde{A}|$ from eq. (F.11)

$$
I_{2}^{\mathrm{ren}}=\left\{\frac{\pi}{2} \frac{1}{S(C)}\left[\prod_{k=1}^{3} \frac{\Gamma\left(1-\beta_{k}\right)}{\Gamma\left(\beta_{k}\right)}\right]\right\}\left\{\frac{\pi}{8} \prod_{k=1}^{3}\left[\frac{\beta_{k}^{2} \Gamma\left(\beta_{k}-1\right)}{\Gamma\left(2-\beta_{k}\right)}\right]\right\}
$$

Here

$$
\begin{aligned}
{\left[\prod_{k=1}^{3} \frac{\Gamma\left(1-\beta_{k}\right)}{\Gamma\left(\beta_{k}\right)}\right] \prod_{k=1}^{3}\left[\frac{\beta_{k}^{2} \Gamma\left(\beta_{k}-1\right)}{\Gamma\left(2-\beta_{k}\right)}\right] } & =\prod_{k=1}^{3}\left[\beta_{k}^{2} \frac{\Gamma\left(1-\beta_{k}\right)}{\Gamma\left(2-\beta_{k}\right)} \frac{\Gamma\left(\beta_{k}-1\right)}{\Gamma\left(\beta_{k}\right)}\right] \\
& =\prod_{k=1}^{3}\left[-\left(\frac{\beta_{k}}{1-\beta_{k}}\right)^{2}\right]
\end{aligned}
$$

Thus

$$
I_{2}^{\mathrm{ren}}=-\frac{\pi^{2}}{16 S(C)} \prod_{k=1}^{3}\left(\frac{\beta_{k}}{1-\beta_{k}}\right)^{2}
$$

\subsection{Final result}

Our final results for $I_{1}^{\text {ren }}(7.6)$ and $I_{2}^{\text {ren }}(7.23)$ are symmetric with respect to permutations of parameters $\beta_{1}, \beta_{2}, \beta_{3}$ whereas the intermediate steps of the computation were asymmetric. The symmetry of the final result is a good test of the consistency of our renormalization procedure. In fact, it is a test of the compatibility of our analytical renormalization with $\mathrm{SL}(2, \mathbb{R})$ symmetry discussed in section 5.4. In our case of triangular contours, the compatibility of the renormalization procedure with $\mathrm{SL}(2, \mathbb{R})$ symmetry was demonstrated via the explicit computation of $I_{1}^{\text {ren }}$ and $I_{2}^{\text {ren }}$. In principle, one can prove that our analytical renormalization respects $\mathrm{SL}(2, \mathbb{R})$ symmetry for arbitrary polygons but this is a subject of a separate work devoted to arbitrary polygons. 
Now we insert results $(7.6),(7.23)$ into the basic expression (6.12) for $\left\langle\mathcal{S}_{4}\right\rangle^{\text {ren }}$ :

$$
\begin{aligned}
\left\langle\mathcal{S}_{4}\right\rangle^{\mathrm{ren}} & =-\frac{1}{\sigma} \frac{D-2}{(8 \pi)^{2}}\left[I_{1}^{\mathrm{ren}}+\frac{2}{9}(D-2) I_{2}^{\mathrm{ren}}\right] \\
& =-\frac{1}{\sigma} \frac{D-2}{(8 \pi)^{2}}\left[\frac{\pi^{2}}{3 S(C)} \prod_{k=1}^{3} \frac{\beta_{k}}{1-\beta_{k}}-\frac{2}{9}(D-2) \frac{\pi^{2}}{16 S(C)} \prod_{k=1}^{3}\left(\frac{\beta_{k}}{1-\beta_{k}}\right)^{2}\right] \\
& =-\frac{1}{\sigma S(C)} \frac{D-2}{192}\left[\prod_{k=1}^{3} \frac{\beta_{k}}{1-\beta_{k}}-\frac{D-2}{24}\left(\prod_{k=1}^{3} \frac{\beta_{k}}{1-\beta_{k}}\right)^{2}\right]
\end{aligned}
$$

Combining this with eq. (2.24), we complete the derivation of our final result (1.19).

Note that in the case of triangles general polygonal constraint (5.5) is enhanced to

$$
0<\beta_{k}<1 .
$$

Obviously $f_{2}(C)$ (1.19) is regular in this region of $\beta_{k}$.

\section{Conclusions}

Using EST, we have computed term $f_{2}(C)$ of the large-size expansion (1.5) for triangular Wilson loops. The result is given by expression (1.19). The success of the calculation is based on the new version of analytical regularization using SC mapping. This regularization can be applied to arbitrary polygons.

\section{Acknowledgments}

I had the luck of starting this work in the stimulating environment formed and guided by L.N. Lipatov and D.I. Diakonov up to their last days. I appreciate the support and the encouragement by V.Yu. Petrov.

\section{A Properties of Laplace determinants}

\section{A.1 Heat-kernel expansion for Laplace operator}

For Laplace operator acting in the region bounded by contour $C$ one has the following heat-kernel expansion [13, 40-44]:

$$
\operatorname{Tr} e^{t \Delta(C) \stackrel{t \rightarrow 0}{=}} \frac{1}{4 \pi t} S(C)-\frac{1}{8 \sqrt{\pi t}} L(C)-\frac{1}{2} \delta(C)+O\left(t^{1 / 2}\right) .
$$

For polygonal contours $C$

$$
\delta(C)=-\sum_{k=1}^{n_{\mathrm{v}}(C)} \xi\left(\theta_{k}\right) .
$$

Here $\theta_{k}$ are interior angles of polygon $C, n_{\mathrm{v}}(C)$ is the number of vertices and

$$
\xi(\theta)=\frac{\pi^{2}-\theta^{2}}{12 \pi \theta} .
$$

For function $\xi(\theta)$, an integral representation was derived by M. Kac in ref. [41]. Explicit expression (A.3) for $\xi(\theta)$ was obtained by D.B. Ray (the derivation is described in ref. [45]). 


\section{A.2 Renormalization of Laplace determinants}

The determinant of Laplace operator in the proper-time regularization

$$
\ln \operatorname{Det}_{\tau}[-\Delta(C)]=-\int_{\tau}^{\infty} \frac{d t}{t} \operatorname{Tr} e^{t \Delta(C)}
$$

has a divergence in the limit $\tau \rightarrow+0$ which is controlled by heat-kernel expansion (A.1):

$$
\ln \operatorname{Det}_{\tau}[-\Delta(C)] \stackrel{\tau \rightarrow++0}{=}-\frac{1}{4 \pi \tau} S(C)+\frac{1}{4 \sqrt{\pi \tau}} L(C)-\frac{1}{2} \delta(C) \ln \tau+O\left(\tau^{0}\right) .
$$

In $\zeta$-regularization method, one first defines the regularizing $\zeta$-function at $\operatorname{Re} s>1$

$$
Z_{C}(s)=\operatorname{Tr}\left[(-\Delta(C)]^{-s}=\frac{1}{\Gamma(s)} \int_{0}^{\infty} d t t^{s-1} \operatorname{Tr} e^{t \Delta(C)} .\right.
$$

Then one performs the analytical continuation in $s$ and computes the derivative

$$
Z_{C}^{\prime}(s) \equiv \frac{d}{d s} Z_{C}(s)
$$

Laplace determinant in the $\zeta$-regularization is given by

$$
\operatorname{Det}_{\zeta}[-\Delta(C)]=\exp \left[-Z_{C}^{\prime}(0)\right]
$$

and has the property

$$
\operatorname{Det}_{\zeta}[-\Delta(\lambda C)]=\lambda^{\delta(C)} \operatorname{Det}_{\zeta}[-\Delta(C)]
$$

where $\delta(C)$ is given by eq. (A.2).

\section{B 1-loop EST corrections}

According to (2.13) terms

$$
f_{\ln }(C) \ln \lambda+f_{0}(C)
$$

of expansion (1.5) arise from the 1-loop EST contribution. These terms are generated by Laplace determinant coming from Gaussian integral (2.19) so that roughly speaking

$$
-\frac{D-2}{2} \ln \operatorname{Det}[-\Delta(\lambda C)] \rightarrow f_{\ln }(C) \ln \lambda+f_{0}(C) .
$$

However, precise expressions are sensitive to certain technical subtleties because both MGT and EST have ultraviolet divergences which must be renormalized in a consistent way matching MGT and EST. The explicit expression for $f_{\ln }(C)$ can be extracted from the naive result (B.1) by comparing eq. (B.1) with eq. (A.9):

$$
f_{\ln }(C)=-\frac{D-2}{2} \delta(C)=\frac{D-2}{2} \sum_{k=1}^{n_{\mathrm{v}}(C)} \xi\left(\theta_{k}\right)
$$

where $\xi(\theta)$ given by eq. (A.3). 
Now let us turn to $f_{0}(C)$. Wilson loop $W(C)$ is not a renormalization invariant quantity so that the single functional $f_{0}(C)$ is not a physical quantity and any attempt to write an explicit expression for single functional $f_{0}(C)$ will result in an ugly combination of scheme dependent parameters. However, simple elegant formulas can be written for linear combinations (2.15) associated with renormalization invariant combinations of Wilson loops — see eqs. (B.13) and (C.29).

The simplest examples of renormalization invariant ratios are combination (1.13) and Creutz ratios

$$
\frac{W\left(L_{1}, L_{2}\right) W\left(L_{1}+L_{1}^{\prime}, L_{2}+L_{2}^{\prime}\right)}{W\left(L_{1}+L_{1}^{\prime}, L_{2}\right) W\left(L_{1}, L_{2}+L_{2}^{\prime}\right)}
$$

made of Wilson loops $W\left(L, L^{\prime}\right)$ for rectangles with sides $L, L^{\prime}$.

The general form of renormalization invariant ratios in the case of polygonal contours $C_{a}$ is

$$
\prod_{a=1}^{N}\left[W\left(C_{a}\right)\right]^{m_{a}}
$$

where parameters $m_{a}$ must obey several conditions. Two constraints have a simple form:

$$
\begin{aligned}
\sum_{a=1}^{N} m_{a} & =0, \\
\sum_{a=1}^{N} m_{a} L\left(C_{a}\right) & =0 .
\end{aligned}
$$

Here $L\left(C_{a}\right)$ is the perimeter of contour $C_{a}$.

In addition, one must obey a vertex-balance condition. Roughly speaking, each vertex angle value must appear in the numerator of (B.4) as many times as in the denominator. In order to make a more careful formulation of this constraint, let us first define $\Theta=\left\{\theta_{A}\right\}$ as a set of all vertex angle values appearing in polygons $C_{a}$ so that each $\theta_{A}$ is included in $\Theta$ only once (whatever often it may appear in polygons $C_{a}$ ):

$$
\theta_{A} \neq \theta_{B} \text { if } A \neq B .
$$

Next, let $p_{a A}$ be the number of occurrences of angle value $\theta_{A}$ among vertices of polygon $C_{a}$ (so that possible values are $p_{a A}=0,1,2, \ldots$ ). Then the vertex-balance constraint reads

$$
\sum_{a=1}^{N} m_{a} p_{a A}=0 .
$$

A practically important equivalent form of vertex-balance constraint (B.8) is

$$
\sum_{a=1}^{N} \sum_{i=1}^{n_{\mathrm{v}}\left(C_{a}\right)} m_{a} \phi\left(\theta_{a i}\right)=0
$$

where $\theta_{a i}$ with $i=1,2,3, \ldots, n_{\mathrm{v}}\left(C_{a}\right)$ are interior angles of polygon $C_{a}$ and $\phi(\theta)$ is an arbitrary function of angle $\theta$. 
Sometimes one also needs area-balance condition

$$
\sum_{a=1}^{N} m_{a} S\left(C_{a}\right)=0
$$

Once all balance conditions (B.5), (B.6), (B.8), (B.10) are satisfied, one finds from eqs. (2.1), (2.12)

$$
\prod_{a=1}^{N}\left[W\left(\lambda C_{a}\right)\right]^{m_{a}} \stackrel{\lambda \rightarrow \infty}{=}\left\{\prod_{a=1}^{N}\left\{\operatorname{Det}\left[-\Delta\left(C_{a}\right)\right]\right\}^{m_{a}}\right\}^{-(D-2) / 2}\left[1+O\left(\lambda^{-2}\right)\right] .
$$

Note that conditions (B.5), (B.6), (B.8), (B.10) guarantee that ultraviolet divergences of MGT cancel on the 1.h.s. of eq. (B.11) whereas ultraviolet divergences of EST cancel on the r.h.s. .

More exactly, the l.h.s. of eq. (B.11) is a renormalization invariant quantity in MGT because renormalization factors associated with perimeter and cusp divergences cancel due to conditions (B.6), (B.9). On the r.h.s., conditions (B.5), (B.6), (B.8), (B.10) guarantee the cancellation of all divergences and the stability of the result with respect to a wide class of renormalization schemes including the renormalizations based on proper-time regularization (A.4) and $\zeta$-regularization (A.8).

If one imposes only constraints (B.5), (B.6), (B.8) but omits area-balance condition (B.10) then eq. (B.11) must be modified. This modification depends on the renormalization scheme for Laplace determinants. In the case of $\zeta$-renormalization (A.8) one has under conditions (B.5), (B.6), (B.8)

$$
\begin{aligned}
& {\left[\exp \left(\lambda^{2} \sigma \sum_{a=1}^{N} m_{a} S\left(C_{a}\right)\right)\right] \prod_{a=1}^{N}\left[W\left(\lambda C_{a}\right)\right]^{m_{a}}} \\
& \stackrel{\lambda \rightarrow \infty}{=}\left\{\prod_{a=1}^{N}\left\{\operatorname{Det}_{\zeta}\left[-\Delta\left(C_{a}\right)\right]\right\}^{m_{a}}\right\}^{-(D-2) / 2}\left[1+O\left(\lambda^{-2}\right)\right] .
\end{aligned}
$$

Here $\sigma$ is the physical string tension of MGT (as it appears in area law (1.1)) and $S\left(C_{a}\right.$ ) is area of polygon $C_{a}$.

Comparing eq. (B.12) with expansion (1.5) and using balance conditions (B.5), (B.6), we find

$$
\sum_{a=1}^{N} m_{a} f_{0}\left(C_{a}\right)=-\frac{D-2}{2} \sum_{a=1}^{N} m_{a} \ln \operatorname{Det}_{\zeta}\left[-\Delta\left(C_{a}\right)\right]
$$

The computation of the r.h.s. is discussed in appendix C.

\section{Calculation of Laplace determinants for polygons}

\section{C.1 2D Laplace determinant for general polygons}

\section{C.1.1 Results of E. Aurell and P. Salomonson}

It is well-known that Laplace determinants in $2 \mathrm{D}$ regions can be computed using the conformal anomaly [46-49]. In order to compute Laplace determinant $\operatorname{Det}(-\Delta(C))$ for 
the region bounded by contour $C$, one has to construct a conformal mapping of this region to some standard region (semiplane or circle). In the case of polygons $C$ this conformal mapping is known as Schwarz-Christoffel (SC) mapping. Thus combining the conformal anomaly and SC mapping, one can compute Laplace determinants for arbitrary polygons. However, on this way one must solve two problems:

- the anomaly-based representation for Laplace determinants has an integral form and this integral must be computed,

- this integral representation has cusp divergences which must be renormalized.

These two problems were successfully solved by E. Aurell and P. Salomonson in ref. [39] where the renormalized functional determinant (in $\zeta$-regularization) of two-dimensional Laplace operator with Dirichlet boundary condition for an arbitrary polygonal region was computed and expressed via parameters of SC mapping.

In this appendix we compute the combinations of Laplace determinants appearing on the r.h.s. of eqs. (B.12), (B.13)

$$
\prod_{a=1}^{N}\left\{\operatorname{Det}_{\zeta}\left[-\Delta\left(C_{a}\right)\right]\right\}^{m_{a}},
$$

using the expressions of ref. [39] for $\operatorname{Det}_{\zeta}\left[-\Delta\left(C_{a}\right)\right]$ and making simplifications based on vertex-balance condition (B.8).

In order to simplify the extraction of final expressions for Laplace determinants from ref. [39], we provide a small dictionary relating the notation used in the current work (l.h.s.) and the notation adopted in ref. [39] (r.h.s. with label AS).

Polygonal boundary $C$ of the region where Laplace operator acts:

$$
C=P_{\mathrm{AS}} .
$$

Area of the region bounded by contour $C$ :

$$
S(C)=A_{\mathrm{AS}} .
$$

Complex coordinate in the polygon plane:

$$
\zeta=z_{\mathrm{AS}} .
$$

(Do not confuse $\zeta$ coordinates with $\zeta$-renormalization.)

Logarithm of Laplace determinant in $\zeta$-renormalization (A.8):

$$
\ln \operatorname{Det}_{\zeta}[-\Delta(C)]=-\left[Z_{P}^{\prime}(0)\right]_{\mathrm{AS}} .
$$

Function $\delta(C)$ given by eqs. (A.2), (A.3):

$$
\delta(C)=-2\left[Z_{P}(0)\right]_{\mathrm{AS}} .
$$

Function $Z_{1-\beta}^{\prime}(0)$ (do not confuse it with quantity $Z_{P}^{\prime}(0)$ appearing in eq. (C.5)) playing an important role in ref. [39] but irrelevant for our work:

$$
h(\beta)=\left[Z_{1-\beta}^{\prime}(0)\right]_{\mathrm{AS}} .
$$

For angles of polygons ref. [39] uses the same parameters $\theta_{k}, \beta_{k}, \alpha_{k}$ as in this work (but with Greek indices) — see eqs. (5.2), (5.7). 


\section{C.1.2 SC mapping from unit circle to polygon}

One should keep in mind that ref. [39] uses Schwarz-Christoffel (SC) mapping of the unit circle $|u| \leq 1$ in complex $u$-plane to the polygon in complex $\zeta$-plane

$$
\frac{d \zeta}{d u}=e^{\lambda_{0}} \prod_{k=1}^{n_{\mathrm{v}}}\left(u-e^{i \phi_{k}}\right)^{-\beta_{k}}
$$

(the original notation of ref. [39] uses $z$ instead of $\zeta$ ). Here $e^{i \phi_{k}}$ are points on the boundary of the unit circle which are mapped to vertices $\zeta_{k}$ of polygon $C . \lambda_{0}$ is a real constant controlling the size of the polygon.

\section{C.1.3 Result for Laplace determinant}

The general result for the determinant of Laplace operator defined in polygon $C$ with Dirichlet boundary condition in $\zeta$-regularization (A.6)-(A.8) is given by eq. (62) of ref. [39]:

$$
\begin{aligned}
-\ln \operatorname{Det}_{\zeta}[-\Delta(C)]= & \sum_{j=1}^{n_{\mathrm{v}}} h\left(\beta_{j}\right)+\frac{\lambda_{0}}{12} \sum_{j=1}^{n_{\mathrm{v}}} \frac{\left(2-\beta_{j}\right) \beta_{j}}{1-\beta_{j}} \\
& -\frac{1}{12} \sum_{1 \leq j \neq k \leq n_{\mathrm{v}}} \frac{\beta_{j} \beta_{k}}{1-\beta_{j}} \ln \left|e^{i \phi_{j}}-e^{i \phi_{k}}\right| .
\end{aligned}
$$

The r.h.s. contains circular SC parameters $\lambda_{0},\left\{\phi_{k}\right\},\left\{\beta_{k}\right\}$ and function $h(\beta)$ (C.7) whose explicit form plays no role for our work.

\section{C.1.4 SC mapping: from unit circle to semiplane}

One can easily establish a connection between semiplane version of SC mapping (5.1) and unit-circle version of SC mapping (C.8). Indeed, one can map the unit circle in the $u$-plane to the upper semiplane of complex variable $z$ using linear-fractional transformation

$$
z=i \frac{1-u}{1+u}
$$

with the inverse mapping

$$
u=\frac{1+i z}{1-i z} .
$$

Transformation (C.10) maps points $e^{i \phi_{k}}$ on the boundary of the unit circle in the $u$-plane to points $z_{k}$ on the real axis of the $z$-plane

$$
\begin{aligned}
z_{k} & =\tan \left(\frac{1}{2} \phi_{k}\right), \\
e^{i \phi_{k}} & =\frac{1+i z_{k}}{1-i z_{k}} .
\end{aligned}
$$

Defining

$$
A=\frac{1}{2} e^{\lambda_{0}}\left[-i \exp \left(\frac{i}{2} \sum_{j=1}^{n_{\mathrm{v}}} \beta_{j} \phi_{j}\right)\right]\left[\prod_{j=1}^{n_{\mathrm{v}}}\left[\cos \left(\frac{1}{2} \phi_{j}\right)\right]^{-\beta_{j}}\right],
$$

one finds that in terms of the $z$-semiplane parametrization, SC mapping (C.8) takes form (5.1). 


\section{C.1.5 Laplace determinants in terms of semiplane parameters of SC mapping}

Using relation (C.12), we find

$$
\ln \left|e^{i \phi_{j}}-e^{i \phi_{k}}\right|=\ln \left|z_{k}-z_{j}\right|+\ln \left|\cos \left(\frac{1}{2} \phi_{j}\right)\right|+\ln \left|\cos \left(\frac{1}{2} \phi_{k}\right)\right|+\ln 2 .
$$

It follows from eq. (C.14) that

$$
\lambda_{0}=\ln |A|+\ln 2+\sum_{j=1}^{n_{\mathrm{v}}} \beta_{j} \ln \left|\cos \left(\frac{1}{2} \phi_{j}\right)\right| .
$$

Combining the last two equations and using constraint (5.6), one can derive relation

$$
\begin{aligned}
& \lambda_{0} \sum_{j=1}^{n_{\mathrm{v}}} \frac{\left(2-\beta_{j}\right) \beta_{j}}{1-\beta_{j}}-\sum_{1 \leq j \neq k \leq n_{\mathrm{v}}} \frac{\beta_{j} \beta_{k}}{1-\beta_{j}} \ln \left|e^{i \phi_{j}}-e^{i \phi_{k}}\right| \\
& =-\sum_{1 \leq j \neq k \leq n_{\mathrm{v}}} \frac{\beta_{j} \beta_{k}}{1-\beta_{j}} \ln \left|\frac{z_{j}-z_{k}}{A}\right| .
\end{aligned}
$$

Now eq. (C.9) takes the form

$$
\ln \operatorname{Det}_{\zeta}[-\Delta(C)]=-\sum_{j=1}^{n_{\mathrm{v}}} h\left(\beta_{j}\right)+\frac{1}{12} \sum_{1 \leq j \neq k \leq n_{\mathrm{v}}} \frac{\beta_{j} \beta_{k}}{1-\beta_{j}} \ln \left|\frac{z_{j}-z_{k}}{A}\right| .
$$

This provides an expression for Laplace determinant via parameters $z_{k}, \beta_{k}, A$ of SC mapping in semiplane form (5.1). The r.h.s. of (C.18) contains function $h(\beta)$ (C.7) which is computed in ref. [39] but cancels in our final formulas.

\section{C.1.6 Taking one SC vertex to infinity}

Sometimes it is convenient to keep one SC vertex at infinity and to work with SC representation (5.21). Taking the limit $\left|z_{n_{\mathrm{v}}}\right| \rightarrow \infty$ on the r.h.s. eq. (C.18) with constraint (5.20) and using relation (5.22), we find the expression for Laplace determinant in terms of SC mapping (5.21)

$$
\begin{aligned}
\ln \operatorname{Det}_{\zeta}[-\Delta(C)]=-\sum_{j=1}^{n_{\mathrm{v}}} h\left(\beta_{j}\right)+\frac{1}{12}[ & \sum_{1 \leq j \neq k \leq n_{\mathrm{v}}-1} \frac{\beta_{j} \beta_{k}}{1-\beta_{j}} \ln \left|z_{j}-z_{k}\right| \\
& \left.-\left(2+\sum_{j=1}^{n_{\mathrm{v}}} \frac{\beta_{j}}{1-\beta_{j}}\right) \ln |\tilde{A}|\right] .
\end{aligned}
$$

\section{C.1.7 Balanced combinations of Laplace determinants}

Let us split

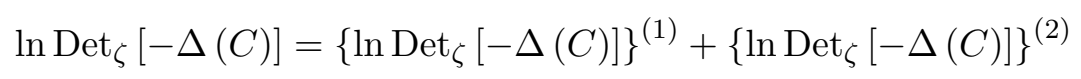

where

$$
\left\{\ln \operatorname{Det}_{\zeta}[-\Delta(C)]\right\}^{(2)}=-\sum_{j=1}^{n_{\mathrm{v}}} h\left(\beta_{j}\right)
$$


Then in the case of SC mapping (5.1) with finite SC vertices we have according to eq. (C.18)

$$
\left\{\ln \operatorname{Det}_{\zeta}[-\Delta(C)]\right\}^{(1)}=\frac{1}{12} \sum_{1 \leq j \neq k \leq n_{\mathrm{v}}} \frac{\beta_{j} \beta_{k}}{1-\beta_{j}} \ln \left|\frac{z_{j}-z_{k}}{A}\right| .
$$

In the case of SC mapping (5.21) with vertex $z_{n_{\mathrm{v}}}$ at infinity we derive from eq. (C.19)

$$
\begin{aligned}
& \left\{\ln \operatorname{Det}_{\zeta}[-\Delta(C)]\right\}^{(1)} \\
& =\frac{1}{12}\left[\sum_{1 \leq j \neq k \leq n_{\mathrm{v}}-1} \frac{\beta_{j} \beta_{k}}{1-\beta_{j}} \ln \left|z_{j}-z_{k}\right|-\left(2+\sum_{j=1}^{n_{\mathrm{v}}} \frac{\beta_{j}}{1-\beta_{j}}\right) \ln |\tilde{A}|\right] .
\end{aligned}
$$

Now we can compute the combination of Laplace determinants appearing on the r.h.s. of eq. (B.13). For each polygon $C_{a}$ appearing in eq. (B.13) we introduce SC mapping (5.1)

$$
\frac{d \zeta}{d z}=A_{a} \prod_{k=1}^{n_{\mathrm{v}}\left(C_{a}\right)}\left(z-z_{a k}\right)^{-\beta_{a k}}
$$

Using decomposition (C.20), we obtain

$$
\begin{aligned}
& \ln \prod_{a=1}^{N}\left\{\operatorname{Det}_{\zeta}\left[-\Delta\left(C_{a}\right)\right]\right\}^{m_{a}}=\sum_{a=1}^{N} m_{a}\left\{\ln _{\operatorname{Det}}\left[-\Delta\left(C_{a}\right)\right]\right\}^{(1)} \\
& +\sum_{a=1}^{N} m_{a}\left\{\ln \operatorname{Det}_{\zeta}\left[-\Delta\left(C_{a}\right)\right]\right\}^{(2)} .
\end{aligned}
$$

Here

$$
\sum_{a=1}^{N} m_{a}\left\{\ln \operatorname{Det}_{\zeta}\left[-\Delta\left(C_{a}\right)\right]\right\}^{(2)}=\sum_{a=1}^{N} m_{a} \sum_{j=1}^{n_{\mathrm{v}}\left(C_{a}\right)} h\left(\beta_{a j}\right)=0
$$

vanishes due to vertex-balance condition (B.9).

Hence

$$
\ln \prod_{a=1}^{N}\left\{\operatorname{Det}_{\zeta}\left[-\Delta\left(C_{a}\right)\right]\right\}^{m_{a}}=\sum_{a=1}^{N} m_{a}\left\{\ln \operatorname{Det}_{\zeta}\left[-\Delta\left(C_{a}\right)\right]\right\}^{(1)} .
$$

On the r.h.s. we can use any of two SC representations (C.22), (C.23) for Laplace determinants. In the case when all $\mathrm{SC}$ vertices are finite for all polygons we find inserting (C.22) into eq. (C.27)

$$
\ln \prod_{a=1}^{N}\left\{\operatorname{Det}_{\zeta}\left[-\Delta\left(C_{a}\right)\right]\right\}^{m_{a}}=\frac{1}{12} \sum_{a=1}^{N} m_{a} \sum_{1 \leq j \neq k \leq n_{\mathrm{v}}\left(C_{a}\right)} \frac{\beta_{a j} \beta_{a k}}{1-\beta_{a j}} \ln \left|\frac{z_{a j}-z_{a k}}{A_{a}}\right| .
$$

Remember that this result was derived assuming vertex-balance condition (B.8).

\section{C.1.8 Result for balanced linear combinations of $f_{0}\left(C_{a}\right)$}

Inserting eq. (C.28) into eq. (B.13), we find

$$
\sum_{a=1}^{N} m_{a} f_{0}\left(C_{a}\right)=-\frac{D-2}{24} \sum_{a=1}^{N} m_{a} \sum_{1 \leq j \neq k \leq n_{\mathrm{v}}\left(C_{a}\right)} \frac{\beta_{a j} \beta_{a k}}{1-\beta_{a j}} \ln \left|\frac{z_{a j}-z_{a k}}{A_{a}}\right| .
$$

This result is derived for the set of polygons $C_{a}$ described by SC mappings (C.24), assuming balance conditions (B.5), (B.6), (B.8). 


\section{C.2 Case of triangles}

\section{C.2.1 Result}

The primary subject of this paper is the computation of 2-loop correction $f_{2}(C)$ for triangles. For completeness it makes sense to compute also 1-loop terms $f_{\ln }(C)$ and $f_{0}(C)$ for triangles. Quantity $f_{\ln }(C)$ is given by simple formula (B.2) which can be used for any polygon including triangles. As for $f_{0}(C)$, we must compute the r.h.s. of eq. (C.28) assuming balance conditions. If all polygons $C_{a}$ are triangles and vertex-balance condition (B.8) holds then one can derive relation

$$
\begin{aligned}
& \ln \prod_{a=1}^{N}\left\{\operatorname{Det}_{\zeta}\left[-\Delta\left(C_{a}\right)\right]\right\}^{m_{a}} \\
& =\frac{1}{24} \sum_{a=1}^{N}\left[\sum_{i=1}^{3}\left(\alpha_{a i}^{-1}-\alpha_{a i}\right)\right]\left[-\ln S\left(C_{a}\right)+\sum_{k=1}^{3} \ln \frac{\Gamma\left(\alpha_{a k}\right)}{\Gamma\left(1-\alpha_{a k}\right)}\right]
\end{aligned}
$$

where in agreement with eq. (5.7)

$$
\alpha_{a i}=\frac{1}{\pi} \theta_{a i}
$$

and $\theta_{a i}$ are interior angles $(i=1,2,3)$ of triangle $C_{a}(a=1,2, \ldots, N) . \Gamma$ is Euler Gamma function. Anomalous dimensional structure of logarithmic term $\ln S\left(C_{a}\right)$ containing area $S\left(C_{a}\right)$ of polygon $C_{a}$ on the r.h.s. of eq. (C.30) makes no problem due to vertex-balance condition (B.9). Eq. (C.30) is derived in appendix C.2.3.

Inserting eq. (C.30) into eq. (B.13), we find for triangles $C_{a}$ obeying balance conditions (B.5), (B.6), (B.8)

$$
\begin{aligned}
\sum_{a=1}^{N} m_{a} f_{0}\left(C_{a}\right)= & -\frac{D-2}{48} \\
& \times \sum_{a=1}^{N}\left[\sum_{i=1}^{3}\left(\alpha_{a i}^{-1}-\alpha_{a i}\right)\right]\left[-\ln S\left(C_{a}\right)+\sum_{k=1}^{3} \ln \frac{\Gamma\left(\alpha_{a k}\right)}{\Gamma\left(1-\alpha_{a k}\right)}\right] .
\end{aligned}
$$

\section{C.2.2 Nontriviality of vertex-balance condition for triangles}

Before deriving relation (C.30) it makes sense to check whether this relation is of any use. The problem is that in the case of triangles the combination of vertex-balance condition (B.8) and angle sum rules (5.4) for each triangle is very restrictive. One can easily obey balance conditions using simplest renormalization invariant combination (1.13) but for the computation of large-size expansion (1.14) of this combination it is sufficient to know function $f_{\ln }(C)$ whereas $f_{0}(C)$ does not appear in (1.14) at all. In other words, for combination (1.13) one has

$$
\begin{aligned}
\ln \prod_{a=1}^{N}\left\{\operatorname{Det}_{\zeta}\left[-\Delta\left(C_{a}\right)\right]\right\}^{m_{a}} & =0, \\
\sum_{a=1}^{N} m_{a} f_{0}\left(C_{a}\right) & =0 .
\end{aligned}
$$


One can wonder whether using only triangle contours $C_{a}$ we can satisfy balance condition in a non-trivial way so that

$$
\sum_{a=1}^{N} m_{a} f_{0}\left(C_{a}\right) \neq 0
$$

i.e. without using similar triangles like in eq. (1.13). The answer to this question is positive but the examples are rather exotic because vertex-balance constraint (B.8) must be combined with the angle sum constraint (5.4)

$$
\theta_{a 1}+\theta_{a 2}+\theta_{a 3}=\pi
$$

for each triangle $C_{a}$.

An example of the solution of this problem can be constructed using Wilson loops $w\left(n_{a 1}, n_{a 2}, n_{a 3}\right)$ for triangles $C_{a}$ with interior angles

$$
\theta_{a k}=\frac{\pi}{9} n_{a k}
$$

with angle sum rule

$$
n_{a 1}+n_{a 2}+n_{a 3}=9 .
$$

One can easily check that combination of Wilson loops

$$
\frac{w(1,3,5)[w(2,3,4)]^{2}}{w(1,4,4) w(2,2,5) w(3,3,3)}
$$

obeys both vertex-balance condition (B.8) and angle sum rule (C.38) for each triangle.

\section{C.2.3 Derivation}

Now we turn to the derivation of eq. (C.30). In principle, one can derive eq. (C.30) from general equation (C.28). On the other hand, one can profit from the explicit expression computed in ref. [39] for Laplace determinant with triangle contour $C$. One can read this explicit expression from eqs. (68) and (69) of ref. [39]. In the original notation of ref. [39] the result reads

$$
Z_{T}^{\prime}(0)=\sum_{i=1}^{3} Z_{\alpha_{i}}^{\prime}(0)+Z_{T}(0) \ln \left[\frac{\pi}{2 A} \prod_{k=1}^{3} \frac{\Gamma\left(\alpha_{k}\right)}{\Gamma\left(1-\alpha_{k}\right)}\right]
$$

where parameters $\alpha_{k}$ are defined by eq. (5.7) and label $T$ stands for triangle. Using relations of appendix C.1.1 (with $P=T$ ), we can translate eq. (C.40) to the notation adopted in the current work:

$$
\operatorname{Det}_{\zeta}[-\Delta(C)]=\left[\frac{\pi}{2 S(C)} \prod_{k=1}^{3} \frac{\Gamma\left(\alpha_{k}\right)}{\Gamma\left(1-\alpha_{k}\right)}\right]^{-\frac{1}{2} \xi(C)} \exp \left[-\sum_{i=1}^{3} h\left(1-\alpha_{k}\right)\right] .
$$

Comparing this with eqs. (C.20), (C.21) and using eqs. (A.2), (A.3), we get rid of irrelevant functions $h\left(1-\alpha_{k}\right)$

$$
\left\{\ln \operatorname{Det}_{\zeta}[-\Delta(C)]\right\}^{(1)}=\frac{1}{24}\left[\sum_{i=1}^{3}\left(\alpha_{i}^{-1}-\alpha_{i}\right)\right] \ln \left[\frac{\pi}{2 S(C)} \prod_{k=1}^{3} \frac{\Gamma\left(\alpha_{k}\right)}{\Gamma\left(1-\alpha_{k}\right)}\right] .
$$


Alternatively one can derive eq. (C.42) by applying general eq. (C.23) to triangular case $n_{\mathrm{v}}=3$, choosing for simplicity $z_{1}=0, z_{2}=1$ and using relation (F.11) between $\tilde{A}$ and $S(C)$.

Next we insert eq. (C.42) into eq. (C.27) and get rid of factors $\pi / 2$ using vertex-balance condition (B.9):

$$
\begin{aligned}
\sum_{a=1}^{N} m_{a}\left\{\ln \operatorname{Det}_{\zeta}\left[-\Delta\left(C_{a}\right)\right]\right\} & =\sum_{a=1}^{N} m_{a}\left\{\ln \operatorname{Det}_{\zeta}\left[-\Delta\left(C_{a}\right)\right]\right\}^{(1)} \\
& =\frac{1}{24} \sum_{a=1}^{N}\left[\sum_{i=1}^{3}\left(\alpha_{a i}^{-1}-\alpha_{a i}\right)\right]\left[-\ln S\left(C_{a}\right)+\sum_{k=1}^{3} \ln \frac{\Gamma\left(\alpha_{a k}\right)}{\Gamma\left(1-\alpha_{a k}\right)}\right] .
\end{aligned}
$$

This completes the derivation of eq. (C.30).

\section{Schwarz derivative}

Schwarz derivative of function $z(w)$ is defined by expression

$$
\{z, w\}=\frac{z^{\prime \prime \prime}}{z^{\prime}}-\frac{3}{2}\left(\frac{z^{\prime \prime}}{z^{\prime}}\right)^{2}
$$

where the prime stands for derivative $d / d w$ :

$$
z^{\prime}=\frac{d z}{d w}, \quad z^{\prime \prime}=\frac{d^{2} z}{d w^{2}}, \quad z^{\prime \prime \prime}=\frac{d^{3} z}{d w^{3}} .
$$

Schwarz derivative has the property

$$
\left\{z_{1}, z_{2}\right\}=-\left(\frac{d z_{1}}{d z_{2}}\right)^{2}\left\{z_{2}, z_{1}\right\}
$$

\section{E Propagator in diagonal limit}

\section{E.1 Green function}

It is well-known that Green function of the two-dimensional Laplace operator in simply connected region $U$ with Dirichlet boundary conditions can be expressed via the conformal mapping of the upper complex semiplane to region $U$. We denote this Laplace operator $\Delta(C)$ where $C$ is the boundary of $U$. Let

$$
x=\tilde{f}(w)
$$

be a representation of this conformal mapping in terms of real coordinates $w^{1}, w^{2}$ on the semiplane and real coordinates $x^{1}, x^{2}$ in region $U$. Then Green function $\left\langle x^{\prime}\left|[\Delta(C)]^{-1}\right| x\right\rangle$ for region $U$ and Green function for the semiplane $\left\langle w^{\prime}\left|\Delta_{\text {semiplane }}^{-1}\right| w\right\rangle$ are connected by relation

$$
\left\langle x^{\prime}\left|[\Delta(C)]^{-1}\right| x\right\rangle=\left\langle w^{\prime}\left|\Delta_{\text {semiplane }}^{-1}\right| w\right\rangle .
$$


Green function for the semiplane can be constructed by the well-known image method from the Green function on the plane:

$$
\left\langle w^{\prime}\left|\Delta_{\text {semiplane }}^{-1}\right| w\right\rangle=\frac{1}{2 \pi}\left[\ln \left|w-w^{\prime}\right|-\ln \left|w^{R}-w^{\prime}\right|\right] .
$$

Here $w^{R}$ is the reflection of point $w$ with respect to $w^{1}$-axis:

$$
\begin{aligned}
& \left(w^{R}\right)^{1}=w^{1}, \\
& \left(w^{R}\right)^{2}=-w^{2} .
\end{aligned}
$$

Hence

$$
\left\langle x^{\prime}\left|[\Delta(C)]^{-1}\right| x\right\rangle=\frac{1}{2 \pi}\left[\ln \left|w-w^{\prime}\right|-\ln \left|w^{R}-w^{\prime}\right|\right] .
$$

This result can be rewritten using complex coordinates

$$
z=w^{1}+i w^{2}
$$

on the upper semiplane of complex $z$ and

$$
\zeta=x^{1}+i x^{2}
$$

for region $U$. Then

$$
\left\langle\zeta^{\prime}\left|[\Delta(C)]^{-1}\right| \zeta\right\rangle=\frac{1}{4 \pi} \ln \frac{\left(z^{\prime}-z\right)\left(z^{*}-z^{*}\right)}{\left(z^{\prime}-z^{*}\right)\left(z^{\prime *}-z\right)}
$$

where

$$
\begin{aligned}
\zeta & =f(z), \\
\zeta^{\prime} & =f\left(z^{\prime}\right)
\end{aligned}
$$

and $f$ is the conformal mapping from the semiplane to $U$ corresponding to mapping $\tilde{f}$ (E.1) formulated in terms of points with real coordinates.

Let

$$
z=\phi(\zeta)
$$

be the inverse mapping. Then eq. (E.9) takes the form

$$
\left\langle\zeta^{\prime}\left|[\Delta(C)]^{-1}\right| \zeta\right\rangle=\frac{1}{4 \pi} \ln \frac{\left[\phi\left(\zeta^{\prime}\right)-\phi(\zeta)\right]\left[\phi\left(\zeta^{\prime}\right)-\phi(\zeta)\right]^{*}}{\left\{\phi\left(\zeta^{\prime}\right)-[\phi(\zeta)]^{*}\right\}\left\{\left[\phi\left(\zeta^{\prime}\right)\right]^{*}-\phi(\zeta)\right\}} .
$$

\section{E.2 Limit 1}

Differentiating eq. (E.13), we find

$$
\begin{aligned}
\frac{\partial}{\partial \zeta^{\prime}}\left\langle\zeta^{\prime}\left|[\Delta(C)]^{-1}\right| \zeta\right\rangle & =\frac{1}{4 \pi} \frac{\partial}{\partial \zeta^{\prime}} \ln \frac{\phi\left(\zeta^{\prime}\right)-\phi(\zeta)}{\phi\left(\zeta^{\prime}\right)-[\phi(\zeta)]^{*}} \\
& =\frac{1}{4 \pi}\left[\frac{\frac{d}{d \zeta^{\prime}} \phi\left(\zeta^{\prime}\right)}{\phi\left(\zeta^{\prime}\right)-\phi(\zeta)}-\frac{\frac{d}{d \zeta^{\prime}} \phi\left(\zeta^{\prime}\right)}{\phi\left(\zeta^{\prime}\right)-[\phi(\zeta)]^{*}}\right]
\end{aligned}
$$


The second differentiation gives

$$
\begin{aligned}
\frac{\partial}{\partial \zeta} \frac{\partial}{\partial \zeta^{\prime}}\left\langle\zeta^{\prime}\left|[\Delta(C)]^{-1}\right| \zeta\right\rangle & =\frac{1}{4 \pi} \frac{\partial}{\partial \zeta}\left[\frac{\frac{d}{d \zeta^{\prime}} \phi\left(\zeta^{\prime}\right)}{\phi\left(\zeta^{\prime}\right)-\phi(\zeta)}\right] \\
& =\frac{1}{4 \pi} \frac{\left[\frac{d}{d \zeta} \phi(\zeta)\right]\left[\frac{d}{d \zeta^{\prime}} \phi\left(\zeta^{\prime}\right)\right]}{\left[\phi\left(\zeta^{\prime}\right)-\phi(\zeta)\right]^{2}}
\end{aligned}
$$

Next we set

$$
\begin{aligned}
\zeta^{\prime} & =\zeta+\eta \\
{\left[\frac{\partial}{\partial \zeta} \frac{\partial}{\partial \zeta^{\prime}}\left\langle\zeta^{\prime}\left|[\Delta(C)]^{-1}\right| \zeta\right\rangle\right]_{\zeta^{\prime}=\zeta+\eta} } & =\frac{1}{4 \pi} \frac{\phi^{\prime}(\zeta) \phi^{\prime}(\zeta+\eta)}{[\phi(\zeta+\eta)-\phi(\zeta)]^{2}}
\end{aligned}
$$

and expand the r.h.s. in powers of $\eta$

$$
\begin{aligned}
\frac{\phi^{\prime}(\zeta) \phi^{\prime}(\zeta+\eta)}{[\phi(\zeta+\eta)-\phi(\zeta)]^{2}} & =\frac{\left[\phi^{\prime}(\zeta)\right]^{2}+\eta \phi^{\prime}(\zeta) \phi^{\prime \prime}(\zeta)+\frac{1}{2} \eta^{2} \phi^{\prime}(\zeta) \phi^{\prime \prime \prime}(\zeta) \ldots}{\eta^{2}\left[\phi^{\prime}(\zeta)+\frac{1}{2} \eta \phi^{\prime \prime}(\zeta)+\frac{1}{6} \eta^{2} \phi^{\prime \prime \prime}(\zeta)+\ldots\right]^{2}} \\
& =\frac{1}{\eta^{2}}+\frac{1}{6}\left[\frac{\phi^{\prime \prime \prime}}{\phi^{\prime}}-\frac{3}{2}\left(\frac{\phi^{\prime \prime}}{\phi^{\prime}}\right)^{2}\right]_{\zeta}+O(\eta)
\end{aligned}
$$

This can be expressed via Schwarz derivative (see eq. (D.1) in appendix D):

$$
\{\phi, \zeta\}=\frac{\phi^{\prime \prime \prime}}{\phi^{\prime}}-\frac{3}{2}\left(\frac{\phi^{\prime \prime}}{\phi^{\prime}}\right)^{2} \text {. }
$$

Hence

$$
\left[\frac{\partial}{\partial \zeta} \frac{\partial}{\partial \zeta^{\prime}}\left\langle\zeta^{\prime}\left|[\Delta(C)]^{-1}\right| \zeta\right\rangle\right]_{\zeta^{\prime}=\zeta+\eta}=\frac{1}{4 \pi}\left(\frac{1}{\eta^{2}}+\frac{1}{6}\{\phi, \zeta\}\right)+O(\eta) .
$$

Since $\phi$ describes conformal mapping $z=\phi(\zeta)$, we can identify

$$
\{\phi, \zeta\}=\{z, \zeta\}
$$

so that

$$
\lim _{\eta \rightarrow 0}\left\{\left[\frac{\partial}{\partial \zeta} \frac{\partial}{\partial \zeta^{\prime}}\left\langle\zeta^{\prime}\left|[\Delta(C)]^{-1}\right| \zeta\right\rangle\right]_{\zeta^{\prime}=\zeta+\eta}-\frac{1}{4 \pi} \frac{1}{\eta^{2}}\right\}=\frac{1}{24 \pi}\{z, \zeta\} .
$$

Combining this with eq. (4.22), we obtain eq. (4.24).

\section{E.3 Limit 2}

Applying derivative $\partial / \partial \zeta^{*}$ to eq. (E.14), we find

$$
\begin{aligned}
\frac{\partial}{\partial \zeta^{*}} \frac{\partial}{\partial \zeta^{\prime}}\left\langle\zeta^{\prime}\left|[\Delta(C)]^{-1}\right| \zeta\right\rangle & =\frac{1}{4 \pi} \frac{\partial}{\partial \zeta^{*}}\left[-\frac{\frac{d}{d \zeta^{\prime}} \phi\left(\zeta^{\prime}\right)}{\phi\left(\zeta^{\prime}\right)-[\phi(\zeta)]^{*}}\right] \\
& =-\frac{1}{4 \pi} \frac{\frac{d}{d \zeta^{\prime}} \phi\left(\zeta^{\prime}\right)\left[\frac{d}{d \zeta^{\prime}} \phi(\zeta)\right]^{*}}{\left[\phi(\zeta)-[\phi(\zeta)]^{*}\right]^{2}} .
\end{aligned}
$$


Taking the limit $\zeta^{\prime} \rightarrow \zeta$, we obtain

$$
\begin{aligned}
\lim _{\zeta^{\prime} \rightarrow \zeta}\left[\frac{\partial}{\partial \zeta^{*}} \frac{\partial}{\partial \zeta^{\prime}}\left\langle\zeta^{\prime}\left|[\Delta(C)]^{-1}\right| \zeta\right\rangle\right] & =-\frac{1}{4 \pi} \frac{\left|\phi^{\prime}(\zeta)\right|^{2}}{\left[\phi(\zeta)-[\phi(\zeta)]^{*}\right]^{2}} \\
& =-\frac{1}{4 \pi}\left|\frac{d z}{d \zeta}\right|^{2} \frac{1}{\left(z-z^{*}\right)^{2}}=\frac{1}{16 \pi}\left|\frac{d z}{d \zeta}\right|^{2}|\operatorname{Im} z|^{-2}
\end{aligned}
$$

Combining this with eq. (4.23), we derive eq. (4.25).

\section{F SC mapping for triangles}

In this appendix we apply SC equation (5.21) with one vertex at infinity to the case of triangles and derive some useful relations between geometric parameters and SC parameters. The case of triangles corresponds to setting $n_{\mathrm{v}}=3$ in eq. (5.21):

$$
\frac{d \zeta}{d z}=\tilde{A} \prod_{k=1}^{2}\left(z-z_{k}\right)^{-\beta_{k}}
$$

Choosing

$$
z_{1}=0, \quad z_{2}=1
$$

we simplify SC equation (F.1) to

$$
\frac{d \zeta}{d z}=\tilde{A} z^{-\beta_{1}}(z-1)^{-\beta_{2}} .
$$

The length of the triangle side SC-mapped to real interval

$$
\left[z_{1}, z_{2}\right]=[0,1]
$$

equals

$$
\begin{aligned}
L_{12} & =\int_{0}^{1} d z\left|\frac{d \zeta}{d z}\right|=|\tilde{A}| \int_{0}^{1} d z z^{-\beta_{1}}(1-z)^{-\beta_{2}} \\
& =|\tilde{A}| B\left(1-\beta_{1}, 1-\beta_{2}\right)=|\tilde{A}| \frac{\Gamma\left(1-\beta_{1}\right) \Gamma\left(1-\beta_{2}\right)}{\Gamma\left(2-\beta_{1}-\beta_{2}\right)}
\end{aligned}
$$

With $\beta_{3}$ given by eq. (5.22)

$$
\beta_{3}=2-\beta_{1}-\beta_{2}
$$

we find

$$
L_{12}=|\tilde{A}| \frac{\Gamma\left(1-\beta_{1}\right) \Gamma\left(1-\beta_{2}\right)}{\Gamma\left(\beta_{3}\right)} .
$$

Area $S(C)$ of the triangle can be computed using elementary geometry:

$$
\begin{aligned}
S(C) & =\frac{1}{2} L_{12} L_{13} \sin \theta_{1}, \\
\frac{L_{12}}{L_{13}} & =\frac{\sin \theta_{3}}{\sin \theta_{2}} .
\end{aligned}
$$


Here $L_{13}$ is the length of the triangle side opposite to the vertex with angle $\theta_{2}$. Angles $\theta_{k}$ of the triangle are given by eq. (5.2). Now we have

$$
\begin{aligned}
S(C) & =\frac{1}{2} L_{12}^{2} \frac{\sin \theta_{1} \sin \theta_{2}}{\sin \theta_{3}} \\
& =\frac{1}{2}\left[|\tilde{A}| \frac{\Gamma\left(1-\beta_{1}\right) \Gamma\left(1-\beta_{2}\right)}{\Gamma\left(\beta_{3}\right)}\right]^{2} \frac{\sin \pi\left(1-\beta_{1}\right) \sin \pi\left(1-\beta_{2}\right)}{\sin \pi\left(1-\beta_{3}\right)},
\end{aligned}
$$

which can be simplified to

$$
\begin{gathered}
|\tilde{A}|^{-2}=\frac{\pi}{2} \frac{1}{S(C)} \frac{\Gamma\left(1-\beta_{1}\right) \Gamma\left(1-\beta_{2}\right) \Gamma\left(1-\beta_{3}\right)}{\Gamma\left(\beta_{1}\right) \Gamma\left(\beta_{2}\right) \Gamma\left(\beta_{3}\right)} \\
\left(z_{1}=0, z_{2}=1\right) .
\end{gathered}
$$

This result can be easily generalized to the case of arbitrary $z_{1}, z_{2}$ :

$$
S(C)=\frac{\pi}{2}\left[|\tilde{A}|\left|z_{1}-z_{2}\right|^{-\beta_{1}-\beta_{2}+1}\right]^{2} \frac{\Gamma\left(1-\beta_{1}\right) \Gamma\left(1-\beta_{2}\right) \Gamma\left(1-\beta_{3}\right)}{\Gamma\left(\beta_{1}\right) \Gamma\left(\beta_{2}\right) \Gamma\left(\beta_{3}\right)} .
$$

\section{G Calculation of $\Pi_{1}^{(2)}$}

Setting

$$
n=2, \quad z_{1}=0, \quad z=x+i y
$$

in eq. (6.15), we find

$$
\begin{aligned}
& \Pi_{1}^{(2)}\left(\alpha,\left\{\gamma_{1}, \gamma_{2}\right\},\left\{0, z_{2}\right\}\right) \\
& =\int_{\mathbb{C}_{+}} d^{2} z|\operatorname{Im} z|^{\alpha-1}|z|^{\gamma_{1}-\alpha-1}\left|z-z_{2}\right|^{\gamma_{2}-\alpha-1} \\
& =\int_{-\infty}^{\infty} d x \int_{0}^{\infty} d y y^{\alpha-1}\left|x^{2}+y^{2}\right|^{\left(\gamma_{1}-\alpha-1\right) / 2}\left|\left(x-z_{2}\right)^{2}+y^{2}\right|^{\left(\gamma_{2}-\alpha-1\right) / 2} .
\end{aligned}
$$

Integrals of this type appear in various problems of mathematical physics. In appendix B of ref. [50] this integral was computed (in a slightly different notation assuming $z_{2}=1$ ) using advanced techniques including a reduction to higher hypergeometric function ${ }_{3} F_{2}$. Below an elementary calculation of integral (G.2) is sketched.

Using the representation

$$
\frac{1}{D_{1}^{\alpha_{1}} D_{2}^{\alpha_{2}}}=\frac{\Gamma\left(\alpha_{1}+\alpha_{2}\right)}{\Gamma\left(\alpha_{1}\right) \Gamma\left(\alpha_{2}\right)} \int_{0}^{1} d \mu \frac{\mu^{\alpha_{1}-1}(1-\mu)^{\alpha_{2}-1}}{\left[\left(D_{1} \mu+D_{2}(1-\mu)\right]^{\alpha_{1}+\alpha_{2}}\right.}
$$

for the factor

$$
\begin{aligned}
& \left|x^{2}+y^{2}\right|^{\left(\gamma_{1}-\alpha-1\right) / 2}\left|\left(x-z_{2}\right)^{2}+y^{2}\right|^{\left(\gamma_{2}-\alpha-1\right) / 2} \\
& =\frac{\Gamma\left(\alpha+1-\frac{\gamma_{1}+\gamma_{2}}{2}\right)}{\Gamma\left(\frac{\alpha+1-\gamma_{1}}{2}\right) \Gamma\left(\frac{\alpha+1-\gamma_{1}}{2}\right)} \int_{0}^{1} d \mu(1-\mu)^{\frac{\alpha-1-\gamma_{1}}{2}} \mu^{\frac{\alpha-1-\gamma_{2}}{2}} \\
& \quad \times\left\{\left[(1-\mu)\left(x^{2}+y^{2}\right)+\mu\left[\left(x-z_{2}\right)^{2}+y^{2}\right]\right]\right\}^{\frac{\gamma_{1}+\gamma_{2}}{2}-\alpha-1}
\end{aligned}
$$


and changing the order of integrations, we obtain

$$
\begin{aligned}
\Pi_{1}^{(2)}\left(\alpha,\left\{\gamma_{1}, \gamma_{2}\right\},\left\{0, z_{2}\right\}\right)= & \frac{\Gamma\left(\alpha+1-\frac{\gamma_{1}+\gamma_{2}}{2}\right)}{\Gamma\left(\frac{\alpha+1-\gamma_{1}}{2}\right) \Gamma\left(\frac{\alpha+1-\gamma_{2}}{2}\right)} \\
& \times \int_{0}^{1} d \mu(1-\mu)^{\frac{\alpha-1-\gamma_{1}}{2}} \mu^{\frac{\alpha-1-\gamma_{2}}{2}} \int_{0}^{\infty} d y y^{\alpha-1} \int_{-\infty}^{\infty} d x \\
& \times\left\{\left[(1-\mu)\left(x^{2}+y^{2}\right)+\mu\left[\left(x-z_{2}\right)^{2}+y^{2}\right]\right]\right\}^{\frac{\gamma_{1}+\gamma_{2}}{2}-\alpha-1} .
\end{aligned}
$$

This order of integrations reduces the calculation to a chain of simple B-function integrals resulting in

$$
\begin{aligned}
& \int_{0}^{1} d \mu(1-\mu)^{\frac{\alpha-1-\gamma_{1}}{2}} \mu^{\frac{\alpha-1-\gamma_{2}}{2}} \int_{0}^{\infty} d y y^{\alpha-1} \int_{-\infty}^{\infty} d x \\
& \quad \times\left\{\left[(1-\mu)\left(x^{2}+y^{2}\right)+\mu\left[\left(x-z_{2}\right)^{2}+y^{2}\right]\right]\right\}^{\frac{\gamma_{1}+\gamma_{2}}{2}-\alpha-1} \\
& =\frac{\sqrt{\pi}}{2}\left|z_{2}\right|^{\gamma_{1}+\gamma_{2}-1-\alpha} \Gamma\left(\frac{\alpha}{2}\right) \frac{\Gamma\left(\frac{\alpha+1}{2}-\frac{\gamma_{1}+\gamma_{2}}{2}\right)}{\Gamma\left(\alpha+1-\frac{\gamma_{1}+\gamma_{2}}{2}\right)} \frac{\Gamma\left(\frac{\gamma_{1}}{2}\right) \Gamma\left(\frac{\gamma_{2}}{2}\right)}{\Gamma\left(\frac{\gamma_{1}+\gamma_{2}}{2}\right)} .
\end{aligned}
$$

We insert this into eq. (G.5)

$$
\begin{aligned}
& \Pi_{1}^{(2)}\left(\alpha,\left\{\gamma_{1}, \gamma_{2}\right\},\{0, z\}\right) \\
& =\frac{\sqrt{\pi}}{2}\left(z_{2}\right)^{\gamma_{1}+\gamma_{2}-1-\alpha} \Gamma\left(\frac{\alpha}{2}\right) \frac{\Gamma\left(\frac{\gamma_{1}}{2}\right)}{\Gamma\left(\frac{\alpha+1}{2}-\frac{\gamma_{1}}{2}\right)} \frac{\Gamma\left(\frac{\gamma_{2}}{2}\right)}{\Gamma\left(\frac{\alpha+1}{2}-\frac{\gamma_{2}}{2}\right)} \frac{\Gamma\left(\frac{\alpha+1}{2}-\frac{\gamma_{1}+\gamma_{2}}{2}\right)}{\Gamma\left(\frac{\gamma_{1}+\gamma_{2}}{2}\right)} .
\end{aligned}
$$

Introducing notation

$$
\gamma_{3}=(\alpha+1)-\left(\gamma_{1}+\gamma_{2}\right)
$$

we find

$$
\begin{aligned}
& \Pi_{1}^{(2)}\left(\alpha,\left\{\gamma_{1}, \gamma_{2}\right\},\left\{0, z_{2}\right\}\right) \\
& =\left[\frac{\sqrt{\pi}}{2}\left|z_{2}\right|^{-\gamma_{3}} \Gamma\left(\frac{\alpha}{2}\right) \prod_{k=1}^{3} \frac{\Gamma\left(\frac{\gamma_{k}}{2}\right)}{\Gamma\left(\frac{\alpha+1}{2}-\frac{\gamma_{k}}{2}\right)}\right]_{\gamma_{3}=(\alpha+1)-\left(\gamma_{1}+\gamma_{2}\right)} .
\end{aligned}
$$

This result was derived in the region of parameters $\alpha, \gamma_{1}, \gamma_{2}$ where integral (G.2) and other intermediate integrals are convergent. The r.h.s. is a meromorphic function of $\alpha, \gamma_{1}, \gamma_{2}$ in agreement with the general properties of functions $\Pi_{P}^{(n)}$ discussed in section 6.3.

Using translational invariance, we generalize eq. (G.9) to

$$
\begin{aligned}
& \Pi_{1}^{(2)}\left(\alpha,\left\{\gamma_{1}, \gamma_{2}\right\},\left\{z_{1}, z_{2}\right\}\right) \\
& =\left[\frac{\sqrt{\pi}}{2}\left|z_{1}-z_{2}\right|^{-\gamma_{3}} \Gamma\left(\frac{\alpha}{2}\right) \prod_{k=1}^{3} \frac{\Gamma\left(\frac{\gamma_{k}}{2}\right)}{\Gamma\left(\frac{\alpha+1}{2}-\frac{\gamma_{k}}{2}\right)}\right]_{\gamma_{3}=(\alpha+1)-\left(\gamma_{1}+\gamma_{2}\right)} .
\end{aligned}
$$




\section{H Calculation of $\Pi_{P}^{(2)}$}

In this appendix we compute function $\Pi_{P}^{(2)}\left(\alpha,\left\{\gamma_{1}, \gamma_{2}\right\},\left\{z_{1}, z_{2}\right\}\right)$ for the special value of argument $\alpha=1$. For simplicity we choose $z_{1}=0, z_{2}=1$. We will perform a formal calculation ignoring divergences of some intermediate integrals but still leading to the correct final result. A rigorous justification of this formal calculation requires methods whose discussion makes sense in the context of the theory of functions $\Pi_{P}^{(n)}$ with arbitrary $n$, i.e. in the case of the theory of analytical regularization for arbitrary polygons $C$.

We start from the integral over complex plane $\mathbb{C}$

$$
\int_{\mathbb{C}} d^{2} z|z|^{2 A}|z-w|^{2 B}=\pi|w|^{2 A+2 B+2} \frac{\Gamma(A+1) \Gamma(B+1) \Gamma(-A-B-1)}{\Gamma(-A) \Gamma(-B) \Gamma(A+B+2)}
$$

which can be easily computed (e.g., using representation (G.3)). Applying derivatives

$$
\left(\frac{\partial}{\partial w}\right)^{m}\left(\frac{\partial}{\partial w^{*}}\right)^{n}
$$

to identity (H.1), we find

$$
\begin{aligned}
& (-1)^{m+n} \frac{\Gamma(B+1)}{\Gamma(B+1-m)} \frac{\Gamma(B+1)}{\Gamma(B+1-n)} \int_{\mathbb{C}} d^{2} z \frac{|z|^{2 A}|z-w|^{2 B}}{(z-w)^{m}\left(z^{*}-w^{*}\right)^{n}} \\
& =\pi \frac{\Gamma(A+1) \Gamma(B+1) \Gamma(-A-B-1)}{\Gamma(-A) \Gamma(-B) \Gamma(A+B+2)} \frac{\Gamma(A+B+2)}{\Gamma(A+B+2-m)} \frac{\Gamma(A+B+2)}{\Gamma(A+B+2-n)} \\
& \quad \times w^{A+B+1-m}\left(w^{*}\right)^{A+B+1-n}
\end{aligned}
$$

Replacing integration variable $z \rightarrow z-w$ and exchanging $A \leftrightarrow B$, we obtain after simplifications:

$$
\begin{aligned}
& \int_{\mathbb{C}} d^{2} z|z|^{2 A}|z-w|^{2 B} z^{m}\left(z^{*}\right)^{n} \\
& =-\pi \frac{\sin (\pi A)}{\sin [\pi(A+B)]} \frac{\Gamma(B+1)}{\Gamma(-B)} \frac{\Gamma(A+1+m) \Gamma(A+1+n)}{\Gamma(A+B+2+m) \Gamma(A+B+2+n)} \\
& \quad \times|w|^{2(A+B+1)} w^{m}\left(w^{*}\right)^{n} .
\end{aligned}
$$

Next we set $w=1$ and notice that after the symmetrization $m \leftrightarrow n$ in eq. (H.4) one can reduce the integral over complex plane $\mathbb{C}$ to the integral over upper semiplane $\mathbb{C}_{+}(4.32)$ :

$$
\begin{aligned}
& \int_{\mathbb{C}} d^{2} z|z|^{2 A}|z-1|^{2 B}\left[z^{m}\left(z^{*}\right)^{n}+z^{n}\left(z^{*}\right)^{m}\right] \\
& =2 \int_{\mathbb{C}_{+}} d^{2} z|z|^{2 A}|z-1|^{2 B}\left[z^{m}\left(z^{*}\right)^{n}+z^{n}\left(z^{*}\right)^{m}\right] .
\end{aligned}
$$

Hence

$$
\begin{aligned}
& \frac{1}{2} \int_{\mathbb{C}_{+}} d^{2} z|z|^{2 A}|z-1|^{2 B}\left[z^{m}\left(z^{*}\right)^{n}+z^{n}\left(z^{*}\right)^{m}\right] \\
& \quad=-\frac{\pi}{2} \frac{\sin (\pi A)}{\sin [\pi(A+B)]} \frac{\Gamma(B+1)}{\Gamma(-B)} \frac{\Gamma(A+1+m) \Gamma(A+1+n)}{\Gamma(A+B+2+m) \Gamma(A+B+2+n)} .
\end{aligned}
$$


The l.h.s. can be interpreted as integral (6.15) defining function $\Pi_{P}^{(2)}$ with a special choice of its polynomial argument $P$ :

$$
P_{m n}(z)=\frac{1}{2}\left[z^{m}\left(z^{*}\right)^{n}+z^{n}\left(z^{*}\right)^{m}\right] .
$$

Setting

$$
\begin{aligned}
n & =2, \quad \alpha=1, \\
z_{1} & =0, \quad z_{2}=1
\end{aligned}
$$

in eq. (6.15), we find from eq. (H.6)

$$
\begin{aligned}
\Pi_{P_{m n}}^{(2)}\left(1,\left\{\gamma_{1}, \gamma_{2}\right\},\{0,1\}\right)= & \frac{\pi}{2} \frac{\sin \left(\pi \frac{\gamma_{1}}{2}\right)}{\sin \left(\pi \frac{\gamma_{1}+\gamma_{2}}{2}\right)} \frac{\Gamma\left(\frac{\gamma_{2}}{2}\right)}{\Gamma\left(1-\frac{\gamma_{2}}{2}\right)} \\
& \times \frac{\Gamma\left(\frac{\gamma_{1}}{2}+m\right) \Gamma\left(\frac{\gamma_{1}}{2}+n\right)}{\Gamma\left(\frac{\gamma_{1}+\gamma_{2}}{2}+m\right) \Gamma\left(\frac{\gamma_{1}+\gamma_{2}}{2}+n\right)} .
\end{aligned}
$$

For a general polynomial

$$
P\left(z, z^{*}\right)=\sum_{m n} c_{m n} z^{m}\left(z^{*}\right)^{n}
$$

with symmetric coefficients

$$
c_{m n}=c_{n m}
$$

we derive from eq. (H.10):

$$
\begin{aligned}
\Pi_{P}^{(2)}\left(1,\left\{\gamma_{1}, \gamma_{2}\right\},\{0,1\}\right)= & \frac{\pi}{2} \frac{\sin \left(\pi \frac{\gamma_{1}}{2}\right)}{\sin \left(\pi \frac{\gamma_{1}+\gamma_{2}}{2}\right)} \frac{\Gamma\left(\frac{\gamma_{2}}{2}\right)}{\Gamma\left(1-\frac{\gamma_{2}}{2}\right)} \\
& \times \sum_{m n} c_{m n} \frac{\Gamma\left(\frac{\gamma_{1}}{2}+m\right) \Gamma\left(\frac{\gamma_{1}}{2}+n\right)}{\Gamma\left(\frac{\gamma_{1}+\gamma_{2}}{2}+m\right) \Gamma\left(\frac{\gamma_{1}+\gamma_{2}}{2}+n\right)} .
\end{aligned}
$$

Open Access. This article is distributed under the terms of the Creative Commons Attribution License (CC-BY 4.0), which permits any use, distribution and reproduction in any medium, provided the original author(s) and source are credited.

\section{References}

[1] K.G. Wilson, Confinement of Quarks, Phys. Rev. D 10 (1974) 2445 [InSPIRE].

[2] A.M. Polyakov, Gauge Fields as Rings of Glue, Nucl. Phys. B 164 (1980) 171 [inSPIRE].

[3] G. 't Hooft, A Planar Diagram Theory for Strong Interactions, Nucl. Phys. B 72 (1974) 461 [INSPIRE].

[4] G. Veneziano, Some Aspects of a Unified Approach to Gauge, Dual and Gribov Theories, Nucl. Phys. B 117 (1976) 519 [InSPIRE].

[5] Y. Makeenko and A.A. Migdal, Quantum Chromodynamics as Dynamics of Loops, Nucl. Phys. B 188 (1981) 269 [INSPIRE]. 
[6] A.A. Migdal, Loop Equations and 1/N Expansion, Phys. Rept. 102 (1983) 199 [INSPIRE].

[7] O. Alvarez, The Static Potential in String Models, Phys. Rev. D 24 (1981) 440 [InSPIRE].

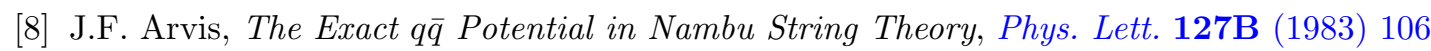
[INSPIRE].

[9] J. Ambjørn and Y. Makeenko, String theory as a Lilliputian world, Phys. Lett. B 756 (2016) 142 [arXiv: 1601.00540] [INSPIRE].

[10] Y. Makeenko, QCD String as an Effective String, in Low dimensional physics and gauge principles: Proceedings, Yerevan, Armenia, Tbilisi, Georgia, 21-29 September 2011, pp. 211-222 (2012) [DOI] [arXiv:1206.0922] [INSPIRE].

[11] Y. Makeenko and P. Olesen, Wilson Loops and QCD/String Scattering Amplitudes, Phys. Rev. D 80 (2009) 026002 [arXiv:0903.4114] [INSPIRE].

[12] Y. Makeenko, Scattering amplitudes of QCD string in the worldline formalism, Phys. Part. Nucl. 45 (2014) 771 [arXiv:1208.1209] [INSPIRE].

[13] M. Lüscher, K. Symanzik and P. Weisz, Anomalies of the Free Loop Wave Equation in the WKB Approximation, Nucl. Phys. B 173 (1980) 365 [InSPIRE].

[14] M. Lüscher, Symmetry Breaking Aspects of the Roughening Transition in Gauge Theories, Nucl. Phys. B 180 (1981) 317 [inSPIRE].

[15] M. Lüscher, G. Munster and P. Weisz, How Thick Are Chromoelectric Flux Tubes?, Nucl. Phys. B 180 (1981) 1 [inSPIRE].

[16] M. Lüscher and P. Weisz, String excitation energies in $\mathrm{SU}(N)$ gauge theories beyond the free-string approximation, JHEP 07 (2004) 014 [hep-th/0406205] [INSPIRE].

[17] H.B. Meyer, Poincaré invariance in effective string theories, JHEP 05 (2006) 066 [hep-th/0602281] [INSPIRE].

[18] O. Aharony and E. Karzbrun, On the effective action of confining strings, JHEP 06 (2009) 012 [arXiv: 0903.1927] [INSPIRE].

[19] O. Aharony and M. Field, On the effective theory of long open strings, JHEP 01 (2011) 065 [arXiv: 1008.2636] [INSPIRE].

[20] O. Aharony and N. Klinghoffer, Corrections to Nambu-Goto energy levels from the effective string action, JHEP 12 (2010) 058 [arXiv: 1008.2648] [INSPIRE].

[21] O. Aharony and M. Dodelson, Effective String Theory and Nonlinear Lorentz Invariance, JHEP 02 (2012) 008 [arXiv: 1111.5758] [INSPIRE].

[22] O. Aharony, M. Field and N. Klinghoffer, The effective string spectrum in the orthogonal gauge, JHEP 04 (2012) 048 [arXiv:1111.5757] [INSPIRE].

[23] O. Aharony and Z. Komargodski, The Effective Theory of Long Strings, JHEP 05 (2013) 118 [arXiv: 1302.6257] [INSPIRE].

[24] M. Billó, M. Caselle, F. Gliozzi, M. Meineri and R. Pellegrini, The Lorentz-invariant boundary action of the confining string and its universal contribution to the inter-quark potential, JHEP 05 (2012) 130 [arXiv:1202.1984] [INSPIRE].

[25] F. Gliozzi and M. Meineri, Lorentz completion of effective string (and p-brane) action, JHEP 08 (2012) 056 [arXiv: 1207.2912] [INSPIRE].

[26] T. Filk, Regularization procedure for string functionals, BONN-HE-81-16 (1981). 
[27] K. Dietz and T. Filk, On the Renormalization of String Functionals, Phys. Rev. D 27 (1983) 2944 [INSPIRE].

[28] M. Billó, M. Caselle, V. Verduci and M. Zago, New results on the effective string corrections to the inter-quark potential, PoS (LATTICE2010) 273 (2010) [arXiv:1012.3935] [INSPIRE].

[29] M. Billó, M. Caselle and R. Pellegrini, New numerical results and novel effective string predictions for Wilson loops, JHEP 01 (2012) 104 [Erratum ibid. 04 (2013) 097] [arXiv:1107.4356] [INSPIRE].

[30] F. Gliozzi, M. Pepe and U.J. Wiese, The Width of the Color Flux Tube at 2-Loop Order, JHEP 11 (2010) 053 [arXiv: 1006.2252] [INSPIRE].

[31] H.B. Meyer, The transverse structure of the QCD string, Phys. Rev. D 82 (2010) 106001 [arXiv: 1008.1178] [INSPIRE].

[32] B.B. Brandt and M. Meineri, Effective string description of confining flux tubes, Int. J. Mod. Phys. A 31 (2016) 1643001 [arXiv:1603.06969] [INSPIRE].

[33] A. Athenodorou, B. Bringoltz and M. Teper, Closed flux tubes and their string description in $D=3+1 \mathrm{SU}(N)$ gauge theories, JHEP 02 (2011) 030 [arXiv:1007.4720] [INSPIRE].

[34] A. Athenodorou, B. Bringoltz and M. Teper, Closed flux tubes and their string description in $D=2+1 \mathrm{SU}(N)$ gauge theories, JHEP 05 (2011) 042 [arXiv:1103.5854] [INSPIRE].

[35] A. Athenodorou and M. Teper, Closed flux tubes in higher representations and their string description in $D=2+1 \mathrm{SU}(N)$ gauge theories, JHEP 06 (2013) 053 [arXiv:1303.5946] [INSPIRE].

[36] A. Athenodorou and M. Teper, Closed flux tubes in $D=2+1 \mathrm{SU}(N)$ gauge theories: dynamics and effective string description, JHEP 10 (2016) 093 [arXiv:1602.07634] [INSPIRE].

[37] A. Athenodorou and M. Teper, $\mathrm{SU}(N)$ gauge theories in $2+1$ dimensions: glueball spectra and k-string tensions, JHEP 02 (2017) 015 [arXiv: 1609.03873] [INSPIRE].

[38] P.V. Pobylitsa, Asymptotic behavior of large polygonal Wilson loops in confining gauge theories, arXiv: 1609.05869 [INSPIRE].

[39] E. Aurell and P. Salomonson, On functional determinants of Laplacians in polygons and simplices, Commun. Math. Phys. 165 (1994) 233.

[40] A. Pleijel, A study of certain Green's functions with applications in the theory of vibrating membranes, Ark. Mat. 2 (1954) 553.

[41] M. Kac, Can one hear the shape of a drum?, Am. Math. Mon. 73 (1966) 1 [InSPIRE].

[42] K. Stewartson and R.T. Waechter, On hearing the shape of a drum: further results, Proc. Cambridge Phil. Soc. 69 (1971) 353.

[43] R. Balian and C. Bloch, Distribution of eigenfrequencies for the wave equation in a finite domain. II. Electromagnetic field. riemannian spaces, Annals Phys. 64 (1971) 271.

[44] P.B. Guilkey, The index theorem and the heat equation, Publish or Perish, Boston (1974).

[45] H.P. McKean and I.M. Singer, Curvature and eigenvalues of the Laplacian, J. Diff. Geom. 1 (1967) 43 [INSPIRE].

[46] A.M. Polyakov, Quantum Geometry of Bosonic Strings, Phys. Lett. 103B (1981) 207 [INSPIRE]. 
[47] B. Durhuus, H.B. Nielsen, P. Olesen and J.L. Petersen, Dual models as saddle point approximations to Polyakov's quantized string, Nucl. Phys. B 196 (1982) 498 [INSPIRE].

[48] B. Durhuus, P. Olesen and J.L. Petersen, Polyakov's Quantized String With Boundary Terms. 2, Nucl. Phys. B 201 (1982) 176 [InSPIRE].

[49] O. Alvarez, Theory of Strings with Boundaries: Fluctuations, Topology and Quantum Geometry, Nucl. Phys. B 216 (1983) 125 [InSPIRE].

[50] A. Belavin and V. Belavin, Higher Equations of Motion in Boundary Liouville Field Theory, JHEP 02 (2010) 010 [arXiv:0911.4597] [INSPIRE]. 